\title{
Extrapolation of Galactic Dust Emission at 100 Microns to Cosmic Microwave Background Radiation Frequencies Using FIRAS
}

\section{Citation}

Finkbeiner, Douglas P., Marc Davis, and David J. Schlegel. 1999. "Extrapolation of Galactic Dust Emission at 100 Microns to Cosmic Microwave Background Radiation Frequencies Using FIRAS." The Astrophysical Journal 524 (2) (October 20): 867-886. doi:10.1086/307852.

\section{Published Version}

$10.1086 / 307852$

\section{Permanent link}

http://nrs.harvard.edu/urn-3:HUL.InstRepos:33462888

\section{Terms of Use}

This article was downloaded from Harvard University's DASH repository, and is made available under the terms and conditions applicable to Other Posted Material, as set forth at http:// nrs.harvard.edu/urn-3:HUL.InstRepos:dash.current.terms-of-use\#LAA

\section{Share Your Story}

The Harvard community has made this article openly available.

Please share how this access benefits you. Submit a story.

\section{Accessibility}


The ASTROPHYSICAL JOURNAL, 524:867-886, 1999 October 20

(C) 1999. The American Astronomical Society. All rights reserved. Printed in U.S.A.

\title{
EXTRAPOLATION OF GALACTIC DUST EMISSION AT 100 MICRONS TO COSMIC MICROWAVE BACKGROUND RADIATION FREQUENCIES USING FIRAS
}

\author{
Douglas P. FinkBeiner AND Marc DAVIS \\ University of California at Berkeley, Departments of Physics and Astronomy, 601 Campbell Hall, Berkeley, CA 94720; dfink@astro.berkeley.edu, \\ marc@deep.berkeley.edu \\ AND \\ DAVID J. SCHLEGEL \\ Princeton University, Department of Astrophysics, Peyton Hall, Princeton, NJ 08544; schlegel@astro.princeton.edu \\ Received 1999 March 5; accepted 1999 June 8
}

\begin{abstract}
We present predicted full-sky maps of submillimeter and microwave emission from the diffuse interstellar dust in the Galaxy. These maps are extrapolated from the $100 \mu \mathrm{m}$ emission and 100/240 $\mu \mathrm{m}$ flux ratio maps that Schlegel, Finkbeiner, \& Davis generated from IRAS and COBE/DIRBE data. Results are presented for a number of physically plausible emissivity models. The correlation of $C O B E /$ FIRAS data with the simple Schlegel, Finkbeiner, \& Davis $\left(v^{2}\right.$ emissivity power law) extrapolation is much tighter than with other common dust templates such as $\mathrm{H}$ I column density or $100 \mu \mathrm{m}$ emission. Despite the apparent success of the Schlegel, Finkbeiner, \& Davis extrapolation, the assumed $v^{2}$ emissivity is inconsistent with the FIRAS data below $800 \mathrm{GHz}$. Indeed, no power-law emissivity function fits the FIRAS data from 200 to $2100 \mathrm{GHz}$. In this paper we provide a formalism for a multicomponent model for the dust emission. A two-component model with a mixture of "silicate" and "carbon-dominated" grains (motivated by Pollack et al.) provides a fit to an accuracy of $\sim 15 \%$ to all the FIRAS data over the entire high-latitude sky. Small systematic differences are found between the atomic and molecular phases of the ISM. COBE/DMR has observed microwave emission that is correlated with thermal dust emission. However, this emission is higher than our model predicts by factors of $1.2,2.4$, and 20 at 90 , 53 , and $31 \mathrm{GHz}$, respectively. This provides evidence that another emission mechanism dominates dust emission at frequencies below $\sim 60 \mathrm{GHz}$. Our predictions for the thermal (vibrational) emission from Galactic dust at $v<3000 \mathrm{GHz}$ are available for general use. These full-sky predictions can be made at the DIRBE resolution of $40^{\prime}$ or at the higher resolution of 6.1 from the Schlegel, Finkbeiner, \& Davis DIRBE-corrected IRAS maps.
\end{abstract}

Subject headings: dust, extinction - infrared: ISM: continuum — submillimeter

\section{INTRODUCTION}

The pioneering Infrared Astronomy Satellite (IRAS) led to the discovery of the ubiquitous infrared cirrus, whose thermal emission is especially visible in the $100 \mu \mathrm{m}$ band (Low et al. 1984). This cirrus, with a characteristic temperature of $\sim 20 \mathrm{~K}$, arches across the sky in long filamentary chains and is present at all Galactic latitudes. However, IRAS was optimized for the detection of point sources, and its ability to map the diffuse cirrus was less than optimal. Because of calibration drifts and hysteresis effects, the resulting IRAS Sky Survey Atlas (ISSA: Wheelock et al. 1994) images are contaminated by significant striping and poor control of large-scale gradients.

The Diffuse Infrared Background Experiment (DIRBE) on the $C O B E$ satellite is the perfect complement to IRAS. It has relatively low angular resolution $(0.7)$ but superbly controlled zero points and gains. This has led to the generation of a map of the far-infrared sky with unprecedented accuracy and uniformity of coverage. Schlegel, Finkbeiner, \& Davis (1998, hereafter SFD98) created a merged map of the IRAS and DIRBE data with an angular resolution of $6^{\prime}$ and DIRBE-quality calibration. Their full-sky map shows the pervasive extent of the infrared cirrus and has proved successful for estimation of extragalactic reddening. But equally important will be the use of this type of data for estimation of Galactic foreground for the coming generation of CMBR experiments, including MAP and Planck and a host of ground- and balloon-based projects.
In this paper, we consider the use of the SFD98 dust map as a predictor for microwave emission from Galactic dust. The SFD98 map is based solely upon 100-240 $\mu \mathrm{m}(1250$ $3000 \mathrm{GHz}$ ) emission. Extrapolation to microwave frequencies is very sensitive to the details of the composition and emissivity properties of the dust. We show that the $v^{2}$ emissivity assumed by SFD98 is inconsistent with the 100$2100 \mathrm{GHz}$ emission probed by the COBE Far Infrared Absolute Spectrophotometer (FIRAS). We use these FIRAS data to constrain the properties of the dust and show that no power-law emissivity model can consistently explain the full spectral range of the dust emission. However, we find excellent agreement with a two-component model whose components we tentatively refer to as silicate- and carbondominated grains. With this model for the dust emissivity function, extrapolation of Galactic dust emission from 100 $\mu \mathrm{m}$ to lower frequencies is based upon the filtered DIRBE $100 / 240 \mu \mathrm{m}$ color temperature.

In $\S 2$, we discuss the $C O B E$ data sets and the details of comparisons using SFD98. Section 3 explores a variety of one-component dust models, demonstrating that a single power-law emissivity fails to explain the data, as does a broadened temperature distribution. Section 4 explores a family of two-component dust models, in which energy balance and the temperature of the separate components are tightly coupled - one of which achieves excellent agreement with the FIRAS data. Section 5 discusses the robustness of this best model with respect to various ISM 
environments, and $\S 6$ compares our predictions to (DMR) microwave observations, demonstrating that the microwave emission may exceed the predictions of any thermal (vibrational) emission mechanisms. This is perhaps the signature of spinning dust grains emitting electric dipole radiation (Draine \& Lazarian 1998b) or the signature of free-free emission. Summary and conclusions are presented in $\S 7$. Details of the frequency bin choice and recalibration can be found in Appendix A. Appendix B contains details on computational methods, and Appendix $\mathrm{C}$ provides details on data presentation.

\section{DATA SETS}

The COBE (COsmic Background Explorer) satellite consisted of three instruments, DMR (Differential Microwave Radiometer), FIRAS (Far Infrared Absolute Spectrophotometer), and DIRBE (Diffuse Infrared Background Experiment). In this paper we shall compare predictions of dust emission based on DIRBE in the far-infrared with that observed by FIRAS at lower frequencies. In addition, we extend this correlation to still lower frequencies $(31.5,53$, and $90 \mathrm{GHz})$ observed by DMR. Although the DMR fluctuations are dominated by intrinsic CMBR anisotropy, a residual correlation with DIRBE is detectable even at high latitudes.

\subsection{FIRAS Spectra}

The objective of the FIRAS instrument was to compare the cosmic microwave background radiation (CMBR) to an accurate blackbody and to observe the dust and line emission from the Galaxy. It is a polarizing Michelson interferometer (Mather 1982), operated differentially with an internal reference blackbody and calibrated by an external blackbody with an emissivity known to better than one part in $10^{4}$. It covers the wavelength range from 0.1 to $10 \mathrm{~mm}$ $(30-3000 \mathrm{GHz})$ in two spectral channels separated at $\sim 0.5$ $\mathrm{mm}(600 \mathrm{GHz})$. The spectral resolution is $\sim 20 \mathrm{GHz}$. Although the design of the FIRAS experiment was optimized for its very successful measurement of the CMBR spectrum (Fixsen et al. 1996), the instrument also measured the spectrum of the dust emission of our Galaxy (e.g., Fixsen et al. 1996). For the highest frequency channels, the Galactic signal dominates all others.

A flared horn antenna aligned with the $C O B E$ spin axis gives the FIRAS a $7^{\circ}$ field of view. The instrument was cooled to $1.5 \mathrm{~K}$ to reduce its thermal emission and enable the use of sensitive bolometric detectors. The FIRAS ceased to operate when the $C O B E$ supply of liquid helium was depleted on 1990 September 21, by which time it had surveyed the sky 1.6 times.

We use the FIRAS Pass 4 Galactic dust spectra (hereafter FIRAS dust spectra) from which CMBR, zodiacal light, and a FIRB model have been subtracted (Fixsen et al. 1997). The data are presented as 213 spectral bins on the resolution 6 skycube map (6144 pixels on the full sky). ${ }^{1}$ Several Galactic emission lines, such as $\mathrm{C}^{+}(157.7 \mu \mathrm{m})$, have been removed and replaced with interpolated values. For our analyses, we have removed the troublesome frequency bins discussed in Appendix A and have recalibrated the entire FIRAS data set down by $1 \%$. Our analyses make use

\footnotetext{
${ }^{1}$ These data are available on the World Wide Web at http:// www.gsfc.nasa.gov/astro/cobe/.
}

of 123 frequency bins at $100<v<2100 \mathrm{GHz}(140$ $\mu \mathrm{m}<\lambda<3 \mathrm{~mm}$ ). Note that data in the lowest two frequency bins are off the page in some of the figures but are used in the fits.

\subsection{DMR Data}

DMR observed the sky at three frequencies, $31.5,53$, and $90 \mathrm{GHz}$, achieving the first detection of anisotropy in the CMBR (Smoot et al. 1992). In this paper we use the 4-Year DMR Skymaps dated 1995 April 18, which have the monopole and dipole removed. These maps do not influence any of our model fits, but are compared with our predictions in $\S 6$.

Kogut et al. (1996) observed a correlation between Galactic dust and the 31.5 and $53 \mathrm{GHz}$ channels of DMR that is much greater than that expected from any models of thermal (vibrational) emission by dust. Alternative explanations such as spinning dust grains (Draine \& Lazarian 1998b) or spatially correlated free-free emission have been proposed but are not well constrained by existing data (cf. de Oliveira-Costa et al. 1998). We discuss this excess emission in $\S 6$.

\subsection{DIRBE Data and SFD Dust Maps 2.3.1. SFD Emission Map}

SFD98 presented a full-sky $100 \mu \mathrm{m}$ cirrus emission map constructed from both the DIRBE and IRAS/ISSA data sets. The map is well calibrated, zodiacal light-subtracted, Fourier-destriped, and point source-subtracted, with a final resolution of 6.1. Complete descriptions of these maps may be found in SFD98. ${ }^{2}$ For comparisons with FIRAS and DMR, the full resolution of the IRAS/DIRBE map is not required. Instead, we use the 0.7 DIRBE map with zodiacal light removed as described in SFD98, with point sources included. The DIRBE map offers a fair comparison with the high-frequency FIRAS data, in which these sources contribute to the measured flux. The comparison is less appropriate in the low-frequency FIRAS data, where typical FIR-luminous sources are expected to contribute little to the measured millimeter flux. However, the contribution from stars and galaxies to the $100 \mu$ m flux is only $\sim 2 \%$ of the diffuse Galactic emission at high latitudes and relatively less at low latitudes.

\subsubsection{Ratio Map}

We also make use of a DIRBE $100 \mu \mathrm{m} / 240 \mu \mathrm{m}$ color ratio similar to that described in SFD98. Because of the poor signal-to-noise ratio in the $240 \mu \mathrm{m}$ map, SFD98 employed a filtering algorithm to give the minimum variance estimate of the dust temperature in each 1.3 Gaussian beam. In each pixel this filter yields the weighted average of the measured flux and a more robust estimator-in the case of SFD98, the estimator is the $|b|>75^{\circ}$ average flux. The weights are chosen so that the ratio of the filtered maps is the minimum variance estimate of the true flux ratio. The process gives the measured ratio in high $\mathrm{S} / \mathrm{N}$ pixels but recovers the highlatitude average ratio of 0.66 in the limit of low $\mathrm{S} / \mathrm{N}$.

The SFD98 algorithm has the unfortunate effect of suppressing temperature variations at high latitude even when those variations are measurable at a resolution of a few degrees. In the current analysis, the $\mathrm{S} / \mathrm{N}$ of FIRAS in a $7^{\circ}$

\footnotetext{
${ }^{2}$ These data are publicly available via the World Wide Web at http:// astro.berkeley.edu/dust.
} 
beam is sufficiently high that this nonlocal filtering algorithm causes undesirable behavior in model fits. When the DIRBE $100 \mu \mathrm{m}$ and $240 \mu \mathrm{m}$ maps are smoothed to $7^{\circ}$, structure in the ratio appears that is not aligned with the imperfectly subtracted zodiacal plane or other potential artifacts in the maps. Rather, the DIRBE $240 \mu \mathrm{m}$ map exhibits structure, even at very low levels, that is correlated with the FIRAS maps at $240 \mu \mathrm{m}$. Therefore, it is presumed that this structure is of extrasolar origin and should not be discarded as it was in the SFD98 analysis.

In the current paper, we have constructed a new ratio map, $\mathscr{R}$, that retains more temperature information. We use the same weight function $\mathscr{W}$ described in SFD98 (eqs. [8] and [9]). But rather than forcing the map to a high-latitude average at low $\mathrm{S} / \mathrm{N}$, we force it to the local $7^{\circ}$ average. High $\mathrm{S} / \mathrm{N}$ regions are little changed from the previous $R$ map, but large-scale temperature structures are now apparent at high Galactic latitudes that were suppressed before. The temperature correction derived from this ratio map has a 1.3 resolution in high $\mathrm{S} / \mathrm{N}$ regions and is applied to the fullresolution $100 \mu \mathrm{m}$ map, not the smoothed $100 \mu \mathrm{m}$ map. This procedure correctly handles the situation where a compact, high-S/N source is located near a diffuse background with a different color temperature. It should be noted here that the same 13 bright sources listed in SFD98 Table 1 were removed from the DIRBE maps before smoothing, to avoid halo artifacts in the $\mathscr{R}$ map. This change in $\mathscr{R}$ produces only a very modest change in the SFD98 reddening predictions. The largest change to predicted reddenings at high latitude is of order $E(B-V)=0.01 \mathrm{mag}$.

\subsubsection{Cosmic IR Background Removal}

The ratio map and derived temperatures are moderately dependent upon the uncertainties in cosmic infrared background (CIB). The CIB represents the extragalactic signal that is unresolved and isotropic in either the DIRBE or FIRAS instruments. This signal is presumably from highredshift $(z \gtrsim 1)$ dust-enshrouded galaxies, which are only beginning to be resolved with ground-based submillimeter observations (cf. Blain et al. 1999). Detections of the CIB at 140 and $240 \mu \mathrm{m}$ were reported last year by SFD98 and Hauser et al. (1998), and upper limits were reported at 100 $\mu \mathrm{m}$. A more definitive analysis is in preparation by Finkbeiner, Schlegel, \& Davis (1999). We remove the CIB from the DIRBE maps in the same way as SFD98 - as part of the zodiacal light model. By using the zero point of the LeidenDwingeloo H I map, a model including CIB and zodiacal light may be fitted and removed. This is an easier problem than the separation of CIB from zodiacal light, which is unnecessary for this paper. One source of error in this could result if there is significant dust emission correlated with $H \alpha$, and the $H \alpha /$ dust correlation has a different zero point than the $\mathrm{H} \mathrm{I} /$ dust correlation. The sense of this would be to add a constant to both $I_{100}$ and $I_{240}$, causing the derived temperature distribution on the sky to broaden or narrow. In other words, a poor $\mathscr{R}$ would produce FIRAS fit residuals that depend on temperature, which would show up in Figure 4. Lack of a temperature-dependent residual indicates that CIB removal errors have had a negligible effect on our model. The rest of our fit procedure - using correlation slopes at each frequency-ignores an isotropic component by construction, so we conclude that we are unaffected by uncertainty in the CIB.

\subsection{Comparing COBE Data Sets 2.4.1. Beam Shapes}

Comparisons between DIRBE and FIRAS data are made at the FIRAS resolution. The FIRAS beam has a frequencydependent shape that is not well measured. The beam is approximately a $7^{\circ}$ diameter top hat in the highest frequency channels, with power-law wings (measured at 750 $\mathrm{GHz}$ from off-axis measurements of the moon; see $\S 7.9 .4$ of FIRAS Exp. Supp. 1997). The beam shape is closer to Gaussian at lower frequencies, with exponential wings from $5^{\circ}$ to $15^{\circ}$ from the beam center (measured at $90 \mathrm{GHz}$ in the lab; see Fixsen et al. 1994). Because the FIRAS scan strategy averages over 32 to $46 \mathrm{~s}$ interferograms, the beam is smeared by typically 2.3 in approximately lines of constant ecliptic longitude. The pixelization of the FIRAS data on $\sim 3^{\circ}$ pixels introduces another effective smoothing. We approximately match the FIRAS beam by first convolving the DIRBE data with a 7.0 circular tophat, then convolving with a 3.0 circular top hat, then smoothing by 2.3 in ecliptic longitude. We ignore the non-Gaussian beam shapes of the DIRBE instruments since they are sufficiently smaller.

We attempted to match the frequency dependence of the FIRAS beam. The signal in the Galactic plane is sufficiently strong $(\sim 100$ times larger than the median value at 500 $\mathrm{GHz}$ ) that the exact sidelobe profile may be important. At low frequencies, the sidelobes exceed $10^{-3}$ within $8^{\circ}$ of the beam center. However, since the profile has been measured at only two frequencies, it is impossible to model the beam to high accuracy. Therefore, the beam shape uncertainties introduce errors of up to $10 \%$ within $7^{\circ}$ of the plane. Because we exclude the sky within $7^{\circ}$ of the Galactic plane from our analyses for other reasons (see $\S 2.4 .2$ ), we simply ignore the complication of frequency dependence of the FIRAS beam.

\subsubsection{Spatial Mask}

Our analysis is limited to those parts of the sky where the far-infrared emission is expected to be dominated by the diffuse interstellar medium. We create a spatial mask that excludes the Galactic plane below $|b|=7^{\circ}$, the Magellanic Clouds, and $\mathrm{H}$ II regions in Orion and Ophiuchus. In such regions, the SFD temperature map is unreliable owing to confusion limits. These are also the regions where the FIRAS data suffer from poorly understood sidelobe contamination. We also mask $1.3 \%$ of the sky where the FIRAS coverage is missing or incomplete and another $15 \%$ where the FIRAS pixel weight is less than 0.4 (the median value is 0.8 ). The final mask excludes $29 \%$ of the sky from our analyses and is shown as the thin black outlines in Figures 8,9 , and 10 . This mask is used throughout this paper except for the comparison in Figure 1 in which the Galactic plane is included and for the comparisons with DMR shown in $\S 6$. For the DMR comparisons we apply the Goddard "custom cut" mask from the 4 yr DMR data analysis, which excludes $37 \%$ of the sky (Bennett et al. 1996).

\subsubsection{Simple Difference Spectra}

For an overview of the three $C O B E$ data sets, it is useful to plot the DMR, FIRAS, and low-frequency DIRBE data on a single plot. The DMR is a differential instrument, so the mean measurement over the sky is zero in each channel. In order to compare it to FIRAS and DIRBE, we plot the difference between "bright" $\left(I_{900 \mathrm{GHz}}>3.0 \mathrm{MJy} \mathrm{sr}^{-1}\right)$ and "faint" regions of the sky. This method has the additional 


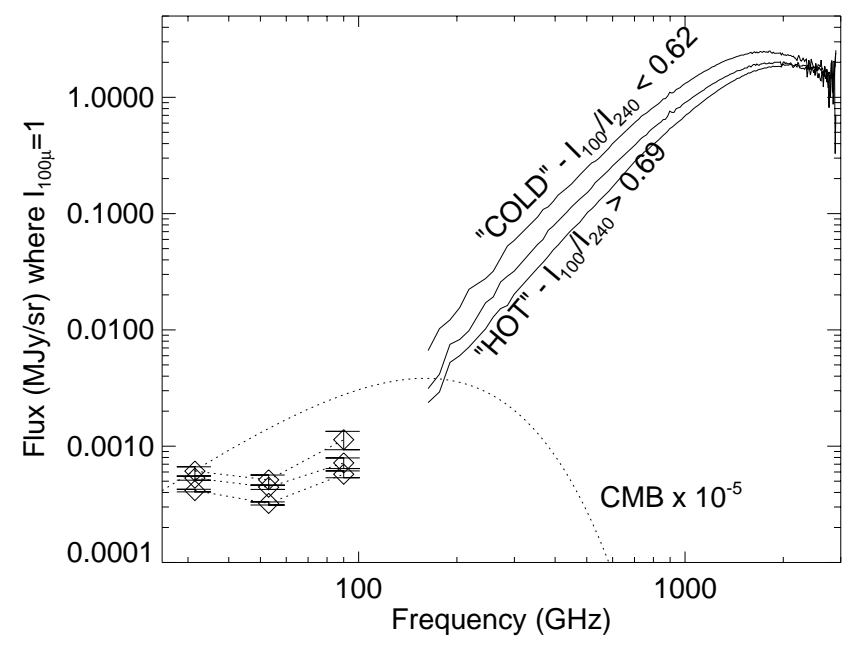

FIG. 1.-Difference spectra from $C O B E$ data, after CMBR monopole and dipole removal. The bright and faint regions of the sky are differenced for each channel in the DMR (diamonds) and FIRAS (solid lines) data sets, excluding the Galactic plane and Magellenic Clouds. The sky is divided into cold, warm, and hot zones based upon DIRBE $I_{100} / I_{240}$ color ratios. The differences in each zone are renormalized to a $100 \mu \mathrm{m}$ flux of $1.0 \mathrm{MJy}$ $\mathrm{sr}^{-1}$, which is a typical flux level for the high-latitude sky. Note the factor of 2 difference between the cold and hot zones at $v \lesssim 700 \mathrm{GHz}$, relative to the $100 \mu \mathrm{m}$ normalization. For comparison, the dotted line represents $10^{-5}$ the level of the CMBR spectrum.

advantage of discarding any isotropic background of cosmic or instrumental origin. We have further divided the sky into cold, warm, and hot zones according to the DIRBE $I_{100} / I_{240}$ color ratio. The cold component $\left(I_{100} / I_{240}<\right.$ $0.62)$ comprises $14 \%$ of the sky, the hot component $\left(I_{100} / I_{240}>0.69\right)$ comprises $26 \%$, and the warm component comprises $44 \%$. The remaining $16 \%$ is masked, rejecting only bad or noisy FIRAS pixels but including signal in the Galactic plane for better $\mathrm{S} / \mathrm{N}$. This plot assumes a monotonic relationship between color ratio and physical temperature but requires no other knowledge of the dust spectra. The difference spectra for these regions are shown in Figure 1. All three curves are renormalized such that the DIRBE $100 \mu \mathrm{m}$ flux is $1 \mathrm{MJy} \mathrm{sr}^{-1}$, a value typical for high-latitude dust. The spectra qualitatively have the correct behavior, with the "cold" regions showing stronger emission at low frequencies relative to $100 \mu \mathrm{m}$.

The FIRAS emission at low frequencies $(200 \lesssim v \lesssim 600$ $\mathrm{GHz}$ ) scales as $\sim v^{3.2}$. Because the Planck function $B_{v}(T)$ approaches $v^{2}$ slowly on the Rayleigh-Jeans tail, the best-fit emission spectrum is $\sim v^{1.7} B_{v}(19 \mathrm{~K})$ over this frequency range, not $\sim v^{1.2} B_{v}(19 \mathrm{~K})$. The temperature $19 \mathrm{~K}$ corre- sponds to the median dust temperature for this fit to the emission spectrum. These considerations indicate that the naive $v^{2}$ emissivity law assumed in SFD98 is incorrect, a matter that will be explored extensively in this paper.

Furthermore, Figure 1 demonstrates that the Galactic emission detected by DMR is inconsistent with any powerlaw extrapolation of the FIRAS data. The DMR 31.5 and $53 \mathrm{GHz}$ channels lie well above the power-law extrapolation of the FIRAS curves. We address this problem in $\S 6$.

\section{ONE-COMPONENT DUST MODELS}

\subsection{Predicted Microwave Emission from SFD98}

A simple but naive prediction for submillimeter/ microwave emission can be made from our previous work. SFD98 extensively studied the emission from dust in the regime $100 \mu \mathrm{m}<\lambda<240 \mu \mathrm{m}$. Assuming a $v^{2}$ emissivity model, the temperature of the dust was mapped with a resolution of 1.3 from the DIRBE $100 \mu \mathrm{m} / 240 \mu \mathrm{m}$ emission ratio, $\mathscr{R}$. The $100 \mu \mathrm{m}$ emission of the dust was mapped with a resolution of 6'.1 by utilizing small-scale information from the IRAS mission. Emission at lower (millimeter/ microwave) frequencies can be predicted by extrapolating the $100 \mu \mathrm{m}$ flux using this temperature fit. For each line of sight in the maps, the emission at frequency $v$ can be expressed as

$$
I_{v}=K_{100}^{-1}(\alpha, T) I_{100} \frac{v^{\alpha} B_{v}(T)}{v_{0}^{\alpha} B_{v_{0}}(T)},
$$

where $B_{v}(T)$ is the Planck function at temperature $T, I_{100}$ is the DIRBE-calibrated $100 \mu \mathrm{m}$ map, $K_{100}(\alpha, T)$ is the color correction factor for the DIRBE $100 \mu \mathrm{m}$ filter when observing a $v^{\alpha} B_{v}(T)$ spectrum, and $v_{0}=3000 \mathrm{GHz}$ is the reference frequency corresponding to $100 \mu \mathrm{m}$. Our values for the color correction factor can be recovered for all values of $\alpha$ used in this paper from the formula

$$
K(\alpha, T)=\frac{\sum_{n} a_{n}(\alpha) \tau^{n}}{\sum_{m} b_{m}(\alpha) \tau^{m}}, \tau \equiv \log _{10} T
$$

where the $a$ and $b$ coefficients may be found in Table 1 .

The choice of an $\alpha=2$ emissivity model was not well motivated in SFD98. The dust column map is only very weakly dependent upon the emissivity law because the entire map is renormalized using direct observations of reddening. Using an $\alpha=1$ emissivity model changes the relative column density of dust between warm and cold regions by only $\sim 1 \%$. However, the extrapolated emission at lower frequencies is highly dependent upon the emissivity of the dust, and the $\alpha=2$ assumption must be tested.

TABLE 1

DIRBE K-CORRECTION FIT COEFFICIENTS

\begin{tabular}{lrrrrrrr}
\hline \hline Coefficient & $\alpha=1.50$ & $\alpha=1.67$ & $\alpha=1.70$ & $\alpha=2.00$ & $\alpha=2.20$ & $\alpha=2.60$ & $\alpha=2.70$ \\
\hline$a_{0} \ldots \ldots \ldots$ & 1.00000 & 1.00000 & 1.00000 & 1.00000 & 1.00000 & 1.00000 & 1.00000 \\
$a_{1} \ldots \ldots \ldots$ & 2.08243 & 2.15146 & 2.14106 & 2.18053 & 2.55941 & 3.16383 & 3.31600 \\
$a_{2} \ldots \ldots \ldots$ & -4.72422 & -4.84539 & -4.83639 & -4.89849 & -5.41290 & -6.23131 & -6.43306 \\
$a_{3} \ldots \ldots \ldots$ & 2.29118 & 2.35210 & 2.35919 & 2.38060 & 2.57867 & 2.86900 & 2.93939 \\
$b_{0} \ldots \ldots \ldots$ & -0.88339 & -0.87985 & -0.93625 & -0.80409 & -0.80318 & -0.50356 & -0.41568 \\
$b_{1} \ldots \ldots \ldots$ & 4.10104 & 4.10909 & 4.19278 & 3.95436 & 4.20361 & 4.07226 & 4.02002 \\
$b_{2} \ldots \ldots \ldots$ & -4.43324 & -4.43404 & -4.46069 & -4.27972 & -4.55598 & -4.70080 & -4.72432 \\
$b_{3} \ldots \ldots \ldots$ & 1.76240 & 1.76591 & 1.77103 & 1.70919 & 1.80207 & 1.87416 & 1.88865 \\
\hline
\end{tabular}

NoTE.-Fit coefficients for DIRBE $100 \mu \mathrm{m}$ band color correction factors $K_{100}(\alpha, T)$, fitted using eq. (2). 


\subsection{Comparison with FIRAS Data at $500 \mathrm{GHz}$}

The extrapolated, millimeter emission from dust as predicted from SFD98 can be compared directly to the FIRAS measurements. As a test of this model, we examine the spatial correlation of FIRAS $500 \mathrm{GHz}$ emission with the DIRBE $100 \mu \mathrm{m}$ map. Such a comparison is most meaningful at a FIRAS frequency that is nearly on the RayleighJeans tail of the dust spectrum but is still easily measured against the $2.73 \mathrm{~K} \mathrm{CMBR}$. This strikes a balance between the poor $\mathrm{S} / \mathrm{N}$ at lower frequencies and the stronger temperature dependence of the spectrum at higher frequencies.

To increase $\mathrm{S} / \mathrm{N}$, we synthesize a broadband FIRAS 500 $\mathrm{GHz}$ channel. We sum the FIRAS measurements in the 10 unmasked channels, $i$, between 400 and $600 \mathrm{GHz}$, weighting by $v^{-3.5}$ to make the summand roughly constant at these frequencies:

$$
\text { FIRAS } 500=\frac{\sum_{i} F_{i} v_{i}^{-3.5}}{\sum_{i} v_{i}^{-3.5}}
$$

The SFD98 extrapolation (eq. [1]) is similarly summed over the same FIRAS frequency bins to generate a predicted broadband flux:

$$
\text { SFD } 500=\frac{\sum_{i} K_{100}^{-1}(\alpha, T) I_{100}\left[v_{i}^{\alpha} B_{v_{i}}(T) / v_{0}^{\alpha} B_{v_{0}}(T)\right] v_{i}^{-3.5}}{\sum_{i} v_{i}^{-3.5}} .
$$

The correlation between FIRAS 500 and SFD 500 is very good (Fig. 2c), with an rms dispersion of $0.2 \mathrm{MJy} \mathrm{sr}^{-1}$ about the best-fit line. For comparison, we plot the correlation with (a) H I column density and (b) DIRBE $100 \mu$ m flux. Both of these show a scatter that is 3.7 times worse than with SFD 500, demonstrating that submillimeter emission from dust is neither simply related to the $\mathrm{H}$ I column density nor is the dust at one temperature everywhere on the sky.

The SFD98 extrapolations work impressively well in predicting $500 \mathrm{GHz}$ emission from dust, despite their assumptions of $v^{2}$ emissivity and one temperature along each line of sight. However, the slope of the regression between FIRAS 500 and our extrapolations differs significantly from unity (formally by nearly $40 \sigma$ ). At lower frequencies, the slope departs even more strongly from unity. This is an indication that a $v^{2}$ emissivity is incorrect for the dust, as was seen from the mean spectrum of large regions of the sky (Fig. 1). This will be addressed in detail in $\S 3.3$.

\subsection{The Spectrum of Dust-correlated Emission}

The many frequency channels of the FIRAS experiment allow detailed comparisons with predictions for the spectrum of dust emission. For each channel of the FIRAS data, we compute a correlation slope with the SFD prediction. The correlation slope is computed as the best-fit slope of the FIRAS column $\left(F_{p}\right)$ versus the predicted column $\left(I_{p}\right)$. By subtracting a weighted mean from each map, the correlation slope, $m$, is insensitive to zero-point uncertainties in either map:

$$
m=\frac{\left\langle W_{p}\left(F_{p}-\bar{F}_{p}\right)\left(I_{p}-\bar{I}_{p}\right)\right\rangle}{\left\langle W_{p}\left(I_{p}-\bar{I}_{p}\right)^{2}\right\rangle},
$$

where $W_{p}$ is the FIRAS pixel weight $\left(W_{p} \sim 1 / \sigma_{p}^{2}\right)$ for pixel $p$. Such a slope is computed for each FIRAS channel centered at $v_{i}$. These slopes are equivalent to that computed in
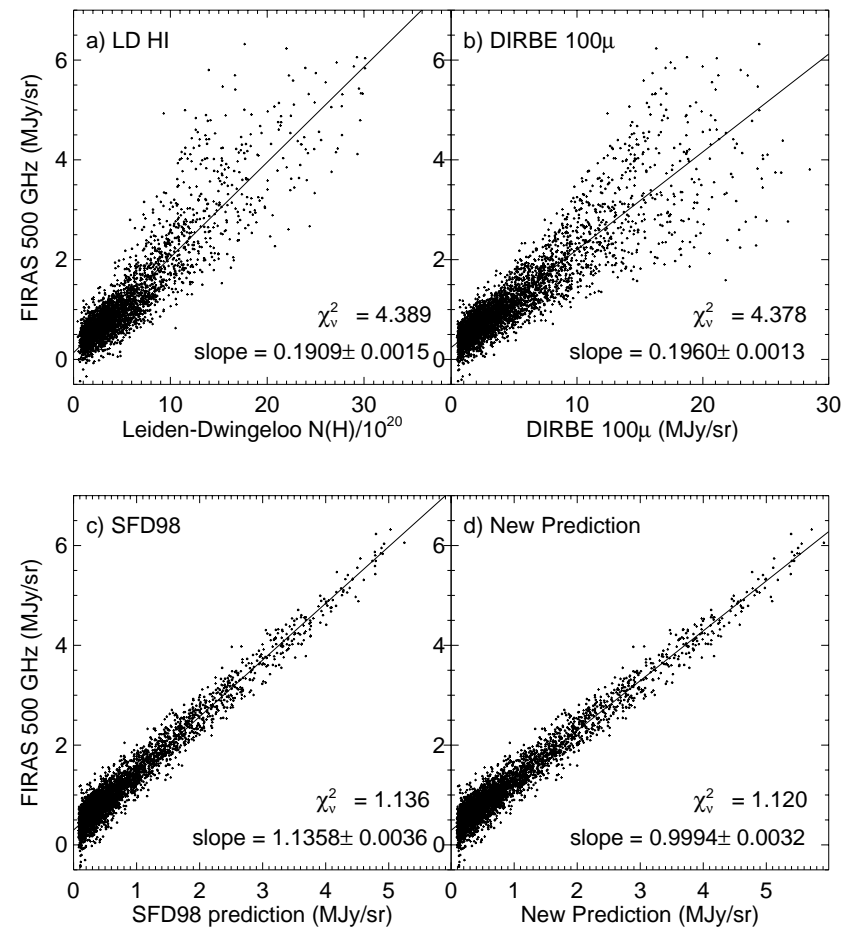

FIG. 2.-FIRAS-DIRBE comparison. Comparison of FIRAS emission in a synthesized $500 \mathrm{GHz}$ broadband vs. (a) H I emission, (b) DIRBE 100 $\mu \mathrm{m}(3000 \mathrm{GHz})$ emission with zodiacal contamination removed, (c) prediction from SFD98 using single-component, $v^{2}$-emissivity model, and $(d)$ prediction from our best-fit two-component model. The comparisons are made over $71 \%$ of the sky. Straight lines are fitted and overplotted using the statistical errors in the FIRAS data. The scatter about this line is $\sim 3.5$ times smaller in $(c)$ or $(d)$ as compared to $(a)$ or $(b)$. The slope in $(d)$ is almost unity, as expected for a good prediction.

Figure $2 c$ for a broadband FIRAS channel. The correlation between the FIRAS column and the SFD prediction is strong and apparently free from systematic errors in all but the lowest frequency channels. For all FIRAS channels $v<2100 \mathrm{GHz}$, we compute $m$ using the $71 \%$ of the sky described in $\S 2.4 .2$. If the $v^{2}$ emissivity model used by SFD98 were valid, the slope would be consistent with unity.

In each panel of Figure 3, the correlation slopes, or "slope spectrum," are plotted as a function of frequency, with the vertical lines extending to $\pm 3 \sigma$. Overplotted in each panel are various models, evaluated for a typical highlatitude $\mathscr{R}$ value of 0.68 . To facilitate comparison with the data, these models are each divided by the same $v^{2}$ prediction as the data. Such a comparison is instructive, but is not used for formal fits, because the ratio of a given model prediction to the $v^{2}$ prediction depends weakly upon $\mathscr{R}$ and therefore varies across the sky. The fitting procedure and exact definition of $\chi^{2}$ is contained in Appendix B. Note that all model ratios are therefore constrained via our temperature correction to be unity at 100 and $240 \mu \mathrm{m}$ (3000 and $1250 \mathrm{GHz}$ ). The data points are also constrained to go through unity at $1250 \mathrm{GHz}$ to the extent that the FIRAS and DIRBE data are consistent.

At $v>500 \mathrm{GHz}$, this slope spectrum is consistent with the $v^{2}$ model to within $10 \%$. At lower frequencies, the slope spectrum increases, demonstrating that there is more emission at low frequencies than a $v^{2}$ emission model would predict. 

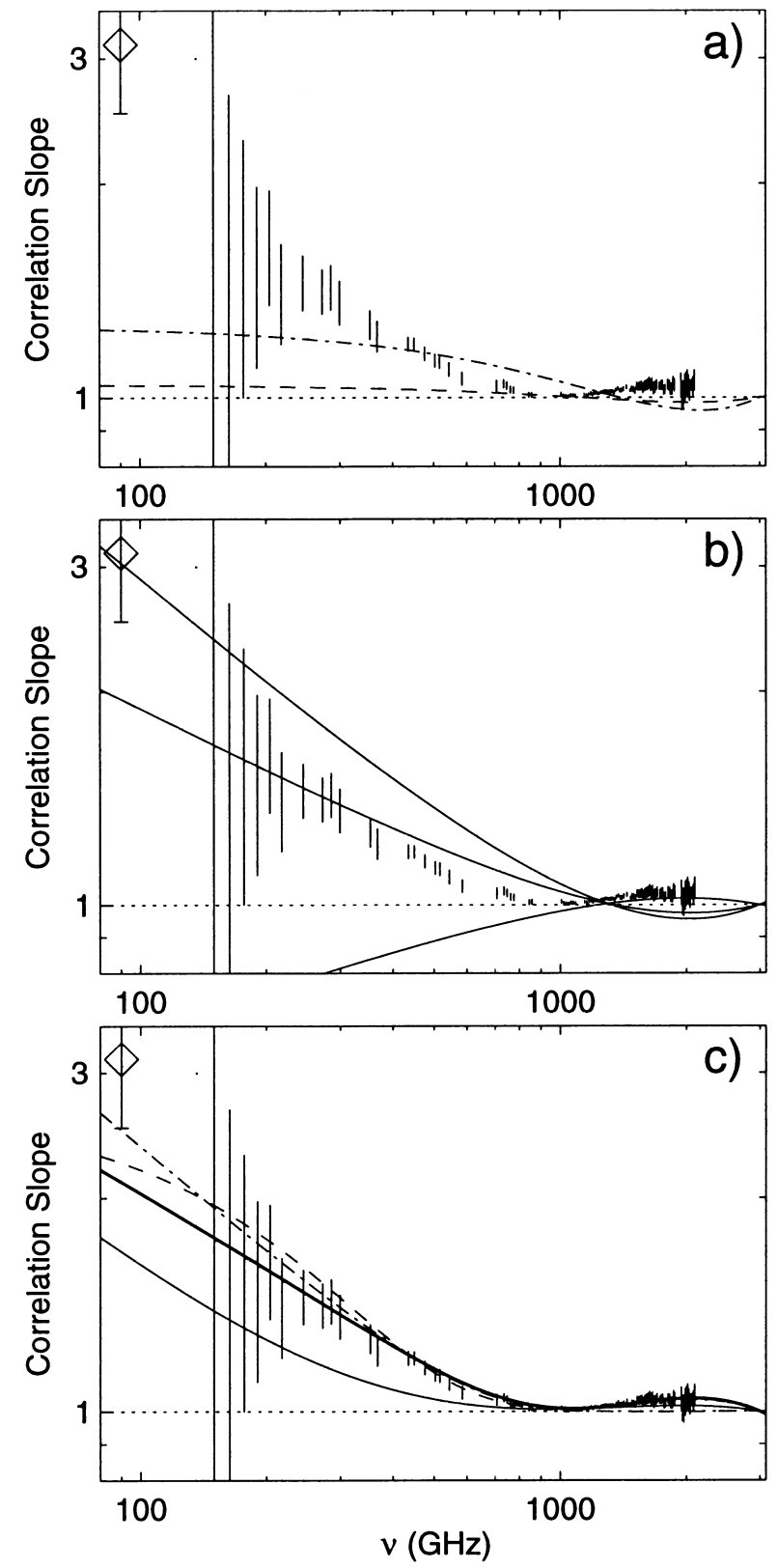

Fig. 3.-Dust-correlated emission, scaled by $v^{2} B_{v}(\bar{T})$ for ease of comparison. The FIRAS data (error bars) would be consistent with unity if the $v^{2}$ emissivity model were correct. Panel $(a)$ overplots broadened temperature models with $\Delta T_{\mathrm{FWHM}}=3 \mathrm{~K}$ (dashed line) and $\Delta T_{\mathrm{FWHM}}=6 \mathrm{~K}$ (dash-dotted line). Panel (b) overplots single-component models with $v^{1.5}$ (top), $v^{1.7}$, and $v^{2.2}$ emssivity laws. The horizontal dotted line corresponds to $v^{2}$. Panel $(c)$ overplots two-component models, with the best-fit model shown as a solid line. See Table 1 for the specific model parameters. These results are not sensitive to an isotropic background in the FIRAS data. The DMR $90 \mathrm{GHz}$ measurement is shown as a diamond. The DMR 30 and 53 $\mathrm{GHz}$ measurements fall well above any model curves.

\subsection{Other Power-Law Emissivities}

There is no power-law emissivity model that fits the FIRAS data. The SFD prediction can be made with other emissivity profiles by modifying the exponent, $\alpha$, in equation (1). An $\alpha=1.5$ emissivity profile results in a better fit at low frequencies but ruins the fit at high frequencies (see Fig. $3 b$ ). An $\alpha=2.2$ emissivity gives a good fit at high frequency but is catastrophically wrong at low frequency. The minimum $\chi^{2}$ is achieved for an $\alpha \approx 2.0$ extrapolation. The value of $\chi^{2}$ more than doubles for $\alpha=1.7$ (see Table 2).

\subsection{Broadened Temperature Distribution}

Our model ignores the possibility of dust temperature variation along a line of sight through the Galaxy. Such a situation may arise from the superposition of different environments with different temperatures, or it may arise from an intrinsic distribution in dust grain sizes and temperatures within a given environment.

The far-IR/submillimeter dust emission is expected to be dominated by large grains $(0.01 \mu \mathrm{m}<a<0.25 \mu \mathrm{m})$, which are in equilibrium with the ambient radiation field. The grains are thought to follow a power law distribution of grain sizes $d n / d a \propto a^{-3.5}$ (Mathis et al. 1977) from about $0.0005 \mu \mathrm{m}$ (the size at which absorption of a single photon can sublimate mass away from the grain) to $0.25 \mu \mathrm{m}$, where the number density appears to fall off based on $R_{V}$ measurements (Kim, Martin, \& Hendry 1994). Since the longwavelength emissivity of a grain scales as its size $a$ times the surface area, or volume $a^{3}$, the larger grains dominate the submillimeter emission (Draine \& Anderson 1985). This will be true unless the slope of the size distribution is steepened to a slope of nearly -4.0 . The very small grains (VSGs; $a \lesssim 0.01 \mu \mathrm{m}$ ) are transiently heated and emit at high effective temperatures for a small fraction of the time but do not contribute significantly to submillimeter emission.

Even for the grains that are large enough to be in equilibrium with the ambient radiation field, there is a slight size-dependent temperature variation. The approximation that the grains are small compared to the wavelength of absorbed radiation is not exactly satisfied at the large end of the grain size distribution, so the larger grains are a bit colder because they absorb less efficiently relative to their emission. For reasonable assumptions about the ISRF, the temperature varies approximately as $a^{-0.06}$ (Draine \& Lee 1984), both for silicate and graphite grains. Over the size range of interest, $0.01 \mu \mathrm{m}<a<0.25 \mu \mathrm{m}$, the temperature range of the grains at a given locale is modest. The dominance of the largest grains' emission results in a narrow distribution of relevant grain temperatures and allows us to use the emission-weighted mean temperature for each component. This approximation is good at $100 \mu \mathrm{m}$ and exact on the Rayleigh-Jeans tail where $I_{v} \propto T$. This greatly simplifies our analysis.

There are two other reasons that the temperature might vary along a line of sight. The cutoff of the grain size distribution at large $a$ might vary, causing the dominant size (and temperature) component to vary along the line of sight. Another possibility is that the interstellar radiation field may vary. Along lines of sight passing through cold molecular clouds, both of these effects should contribute. For extinction predictions (as in SFD98) it is important to understand which of these effects is causing the temperature variation; for extrapolating the emission to microwaves, the cause is unimportant.

In order to model such variations, we experimented with Gaussian-broadened distributions of temperatures with width $\Delta T_{\mathrm{FWHM}}$ along a single line of sight. The ratio of a broadened- $T$ model $\left(\Delta T_{\mathrm{FWHM}}=3 \mathrm{~K}\right)$ to the idealized singletemperature fit is shown as the dashed line in Figure $3 a$. An even broader distribution $\left(\Delta T_{\mathrm{FWHM}}=6 \mathrm{~K}\right)$ is plotted as a dash-dotted line. The broadened- $T$ model changes the predictions by at most $4 \%$ for $\Delta T_{\mathrm{FWHM}}=3 \mathrm{~K}$ and $15 \%$ for $\Delta T_{\mathrm{FWHM}}=6 \mathrm{~K}$. These models retain large and coherent deviations from the FIRAS data. The models are slightly 
TABLE 2

Fit Results fOR DUST EMission Models

\begin{tabular}{|c|c|c|c|c|c|c|c|c|c|c|}
\hline Number & Model & $\alpha_{1}$ & $\alpha_{2}$ & $f_{1}$ & $q_{1} / q_{2}$ & $\left\langle T_{1}\right\rangle$ & $\left\langle T_{2}\right\rangle$ & $P_{1} / P_{2}$ & $\chi^{2}$ & $\chi_{v}^{2}$ \\
\hline $1 \ldots \ldots \ldots$ & One-component: $v^{1.5}$ emis & 1.5 & $\ldots$ & 1.0 & 1.0 & 20.0 & $\ldots$ & $\ldots$ & 24943 & 204 \\
\hline $2 \ldots \ldots \ldots$ & One-component: $v^{1.7}$ emis & 1.7 & $\ldots$ & 1.0 & 1.0 & 19.2 & $\ldots$ & $\ldots$ & 8935 & 73 \\
\hline $3 \ldots \ldots$. & One-component: $v^{2.0}$ emis & 2.0 & $\ldots$ & 1.0 & 1.0 & 18.1 & $\ldots$ & $\ldots$ & 3801 & 31 \\
\hline $4 \ldots \ldots \ldots$ & One-component: $v^{2.2}$ emis & 2.2 & $\ldots$ & 1.0 & 1.0 & 17.4 & $\ldots$ & $\ldots$ & 9587 & 79 \\
\hline $5 \ldots \ldots \ldots$ & Pollack et al. two-component & 1.5 & 2.6 & 0.25 & 0.61 & 17.0 & 17.0 & 0.33 & 1866 & 15.3 \\
\hline $6 \ldots \ldots \ldots$ & Two-component: both $v^{2}$ & 2.0 & 2.0 & 0.00261 & 2480 & 4.9 & 18.1 & 0.0026 & 1241 & 10.3 \\
\hline $7 \ldots \ldots \ldots$ & Two-component: fit $f, q$ & 1.5 & 2.6 & 0.0309 & 11.2 & 9.6 & 16.4 & 0.0319 & 244 & 2.03 \\
\hline $8 \ldots \ldots \ldots$ & Two-component: fit $f, q, \alpha_{1}, \alpha_{2}$ & 1.67 & 2.70 & 0.0363 & 13.0 & 9.4 & 16.2 & 0.0377 & 219 & 1.85 \\
\hline
\end{tabular}

Note.-The dust models are described by $\alpha_{1}, \alpha_{2}, f_{1}$, and $q_{1} / q_{2}$. The mean temperatures for each dust component, $\left\langle T_{1}\right\rangle$ and $\left\langle T_{2}\right\rangle$, are evaluated for the mean $I_{100} / I_{240}$ color ratio in the high-latitude sky. The ratio of power emitted by each component is $P_{1} / P_{2}$. The definition of $\chi^{2}$ is given in $\S \mathrm{B} 3$.

more consistent with the low-frequency FIRAS channels but are less consistent with the high-frequency channels. The value of $\chi^{2}$ for these models is higher than that of the single-temperature $v^{2}$ model. We conclude that a Gaussianbroadened temperature distribution does not fit the data any better than a single-temperature model and would only introduce poorly constrained parameters into our models. The lack of an acceptable one-component model - even when temperature variation along each line of sight is included -indicates the need for multicomponent models, which are discussed in the next section.

\section{MULTICOMPONENT DUST MODELS}

In this section we explain the theoretical motivation for multicomponent dust models, present our general model, and then provide the results for eight specific models.

\subsection{Theoretical Motivation}

The diffuse ISM is known to contain many different types of molecules and dust grains with a broad range of physical properties. In spite of the expected melange of dust grains, it was originally expected that in the far-IR/submillimeter bands, all dust would have similar optical properties. For example, Draine \& Lee (1984) predicted $v^{2}$ emissivity for both silicate and graphite grains.

The emission mechanism corresponding to fundamental vibrations (single photon/phonon interactions) in crystalline dielectric materials is optically inactive due to wavevector conservation, and multiphoton interactions are rare at low temperatures. Therefore, the emissivity of crystalline materials would be expected to be dominated by absorption in the damping wing of an infrared active fundamental vibration, the strength of which goes as $v^{2}$ at low frequencies. In a metallic or semimetallic material, interaction with electrons was expected to dominate FIR absorption, also resulting in $v^{2}$ emissivity (Wooten 1972).

In amorphous materials, the lack of long-range order causes a breakdown of the selection rules that forbid single photon/phonon interactions, and all modes become active. The emissivity power law then depends only upon the density of states, which was also thought to go as $v^{2}$ (Kittel 1976). Thus, amorphous materials were expected to have the same dependence on frequency as other components, but for an entirely different reason. The most notable exception to the $v^{2}$ theory was the case of planar structures such as graphite, which would yield a $v^{1}$ power law by the same reasoning. For an excellent summary of the theoretical details, see Tielens \& Allamandola (1987).
More recent laboratory measurements suggest that universality of $v^{2}$ emissivity is an oversimplification, with different species of grains having differing emissivity laws. The composition and abundance of grains of different species can be constrained by astronomical observations and by observations of solar system bodies. A multicomponent model for interstellar dust has been constructed by Pollack et al. (1994), based on laboratory measurements, observations of molecular cloud cores, and fits to dust shells with temperatures $T \approx 100 \mathrm{~K}$ around young stars. Their model predicts that at frequencies $v \gtrsim 500 \mathrm{GHz}$, dust emission will obey a $v^{2.6}$ emissivity law due to the dominance of carbon species. At lower frequencies, the emission is dominated by astronomical silicates such as olivine $\left([\mathrm{Mg}, \mathrm{Fe}]_{2} \mathrm{SiO}_{4}\right)$ and orthopyroxene $\left([\mathrm{Mg}, \mathrm{Fe}] \mathrm{SiO}_{3}\right)$. This low-frequency prominence of silicates flattens the emissivity profile to $v^{1.5}$ at frequencies $v \lesssim 500 \mathrm{GHz}(\lambda \gtrsim 600 \mu \mathrm{m})$

Despite the complexities in the dust composition, most authors have chosen for simplicity to model the observed emission with a single power-law emissivity. If one's observations are limited to less than a decade in frequency, this parameterization may be adequate to fit the data, especially if one component dominates the emission. However, combining data from all three $C O B E$ instruments results in a tremendous range in observed frequencies. The discussion in $\S 3$ demonstrates that a single power-law emissivity is a poor fit to this combined data. Our physical interpretation is that different grain species dominate the emission at different frequencies.

Laboratory measurements of submillimeter-wave absorption properties of both crystalline and amorphous silicates (Agladze et al. 1996) suggest that $\alpha$ ranges from approximately 1.2 to 2.7 , with some components having a much higher opacity than others (by a factor of $\sim 40$ at 300 $\mathrm{GHz}$ and $20 \mathrm{~K}$ ). These studies motivate a broader search of parameter space.

\subsection{General Multicomponent Model}

We outline a general formalism for describing a mixed population of dust grains. These simple considerations apply only in the limit of large grains $(a>0.01 \mu \mathrm{m})$ which are not transiently heated but instead reach equilibrium with the local radiation field. We neglect emission from very small, transiently heated grains because it is unimportant over the FIRAS frequency range.

\subsubsection{Statement of Model}

Fortunately, for wavelengths $\lambda>100 \mu \mathrm{m}$ the large grains totally dominate the thermal (vibrational) dust emission. 
Since it is common to assume that each component of the dust will have a power-law emissivity over the FIRAS frequency range (Pollack et al. 1994), one can sum these to construct a multicomponent model analogous to equation (1):

$$
I_{p, v}=\frac{\sum_{k} f_{k} Q_{k}(v) B_{v}\left(T_{p k}\right)}{\sum_{k} f_{k} Q_{k}\left(v_{0}\right) B_{v 0}\left(T_{p k}\right) K_{100}\left(\alpha_{k}, T_{p k}\right)} I_{p, 100},
$$

where $f_{k}$ is a normalization factor for the $k$ th component, $T_{p k}$ is the temperature in pixel $p$ of component $k, K_{100}$ is the DIRBE color-correction factor (DIRBE Exp. Supp. 1995), and $I_{p, 100}$ is the SFD98 $100 \mu \mathrm{m}$ flux at pixel $p$ in the DIRBE filter. The emission efficiency $Q(v)$ is the ratio of the emission cross section to the classical cross section of the grain. Because the grains of interest are very small compared to the wavelength of emission, $Q(v) \propto a$, where $a$ is the radius of a spherical grain. One interpretation of this is that the grain is so small that all parts of the grain are close enough to the grain surface to take part in the emission.

The emission opacity (effective area per mass) for a spherical grain of radius $a, \kappa^{\mathrm{em}}(v)$, is related to $Q(v)$ by

$$
\kappa^{\mathrm{em}}(v)=\frac{\pi a^{2}}{\rho V} Q(v)=\frac{3 Q(v)}{4 \rho a} .
$$

Because $Q / a$ is usually taken to be independent of $a$ for $a \ll \lambda$ (cf. Hildebrand 1983), $\kappa^{\mathrm{em}}(v)$ does not depend on grain size. The frequency dependence is taken to be a power law

$$
\kappa^{\mathrm{em}}(v)=\kappa^{\mathrm{em}}\left(v_{0}\right)\left(v / v_{0}\right)^{\alpha_{k}},
$$

where $\alpha_{k}$ is the emissivity index and $\kappa^{\mathrm{em}}\left(v_{0}\right)$ is the opacity of species $k$ at a reference frequency $v_{0}=3000 \mathrm{GHz}$. It will be convenient to interpret $f_{k}$ as the fraction of power absorbed and reemitted by component $k$, so we force the power fractions to sum to unity:

$$
\sum_{k} f_{k}=1
$$

\subsubsection{Temperature Coupling}

The degrees of freedom in this multicomponent model can be substantially reduced by demanding that the components are in equilibrium with the interstellar radiation field (ISRF). If we assume that the ISRF has a constant spectrum everywhere on the sky and varies only in intensity, then we may define $\kappa_{k}^{*}$ to be the effective absorption opacity (cross section per mass) to the ISRF in the limit of low optical depth:

$$
\kappa_{k}^{*}=\frac{\int_{0}^{\infty} \kappa_{k}^{\mathrm{abs}}(v) I_{\mathrm{ISRF}}(v) d v}{\int_{0}^{\infty} I_{\mathrm{ISRF}}(v) d v},
$$

where $I_{\mathrm{ISRF}}(v)$ represents the angle-averaged intensity in the ISRF as a function of frequency and has the same dimensions as $B_{v}$. To avoid confusion, $\kappa_{k}^{\text {abs }}(v)$ designates the optical opacity of component $k$, which is physically related to the submillimeter opacity $\kappa_{k}^{\mathrm{em}}(v)$ but need not be an extension of the power-law expression for $\kappa_{k}^{\mathrm{em}}(v)$ in equation (8). The total power absorbed per mass for species $k$ is given by

$$
U_{k}^{\mathrm{in}}=\kappa_{k}^{*} \int_{0}^{\infty} I_{\mathrm{ISRF}}(v) d v
$$

The power is primarily radiated in the far-infrared and thus is sensitive only to the far-IR emissivity law. The power per mass emitted is

$$
U_{k}^{\text {out }}=\int_{0}^{\infty} \kappa_{k}^{\mathrm{em}}(v) B_{v}\left(T_{k}\right) d v .
$$

Demanding that each grain species is in equilibrium with the ISRF (e.g., $U_{k}^{\text {in }}=U_{k}^{\text {out }}$ ), the energies of all species are related via

$$
\frac{1}{\kappa_{i}^{*}} \int_{0}^{\infty} \kappa_{i}^{\mathrm{em}}(v) B_{v}\left(T_{i}\right) d v=\frac{1}{\kappa_{j}^{*}} \int_{0}^{\infty} \kappa_{j}^{\mathrm{em}}(v) B_{v}\left(T_{j}\right) d v .
$$

Using our parameterization of the emissivities (eq. [8]), we can solve for the temperature of one component as a function of the other:

$$
T_{i}^{4+\alpha_{i}}=\frac{q_{j} Z\left(\alpha_{j}\right)}{q_{i} Z\left(\alpha_{i}\right)}\left(\frac{h v_{0}}{k_{B}}\right)^{\alpha_{i}-\alpha_{j}} T_{j}^{4+\alpha_{j}},
$$

where

$$
q=\kappa^{\mathrm{em}}\left(v_{0}\right) / \kappa^{*}
$$

is essentially the ratio of far-IR emission cross section to the UV/optical absorption cross section, and the integrals are absorbed into the analytic function

$$
Z(\alpha) \equiv \int_{0}^{\infty} \frac{x^{3+\alpha}}{e^{x}-1} d x=\zeta(4+\alpha) \Gamma(4+\alpha) .
$$

Henceforth, we shall use only the ratio of opacities, $q_{k}$, assuming that the dust temperature is sensitive only to this ratio of emission to absorption cross sections. This is not strictly true because $\kappa^{*}$ is weakly dependent upon grain size. However, as we showed in $\S 3.5$, the assumption of a single temperature for each component-and therefore a single $q_{k}$-in each locale is justified.

\subsubsection{Interpretation of $f, q$}

Each dust component is therefore described by three global parameters $\left(f_{k}, q_{k}, \alpha_{k}\right)$ and one parameter that varies with position on the sky, $T_{k}(x)$. Because equation (14) couples the temperature of each component, there is only one independent temperature (e.g., $T_{2}$ ) per line of sight. The interpretation of the $q_{k}$ as IR/optical opacity ratios is obvious, but the meaning of the $f_{k}$ normalization factors is less clear. To understand what the $f_{k}$ are, let us consider the ratio of the power, $P_{i} / P_{j}$, absorbed and reemitted by components $i$ and $j$ :

$$
\frac{P_{i}}{P_{j}}=\frac{f_{i}}{f_{j}} \frac{\int_{0}^{\infty} q_{i}\left(v / v_{0}\right)^{\alpha_{i}} B_{v}\left(T_{i}\right) d v}{\int_{0}^{\infty} q_{j}\left(v / v_{0}\right)^{\alpha_{j}} B_{v}\left(T_{j}\right) d v} .
$$

Combining equations (8), (13), and (15), we see that the integrals are equal:

$$
P_{i} / P_{j}=f_{i} / f_{j} .
$$

Because the $f_{k}$ sum to unity (eq. [9]), we identify $f_{k}$ with the fraction of power absorbed from the ISRF and emitted in the FIR by component $k$. Note that $P_{i} / P_{j}$ is independent of frequency. Note also that $f_{k} / \kappa_{k}^{*}$ is proportional to the mass fraction. Therefore, if the optical opacities of all species were equal (which is unlikely), then $f_{i} / f_{j}$ would measure the mass ratios between species $i$ and $j$.

Whether or not the actual components of the dust physically correspond to these components, equation (6) can be 


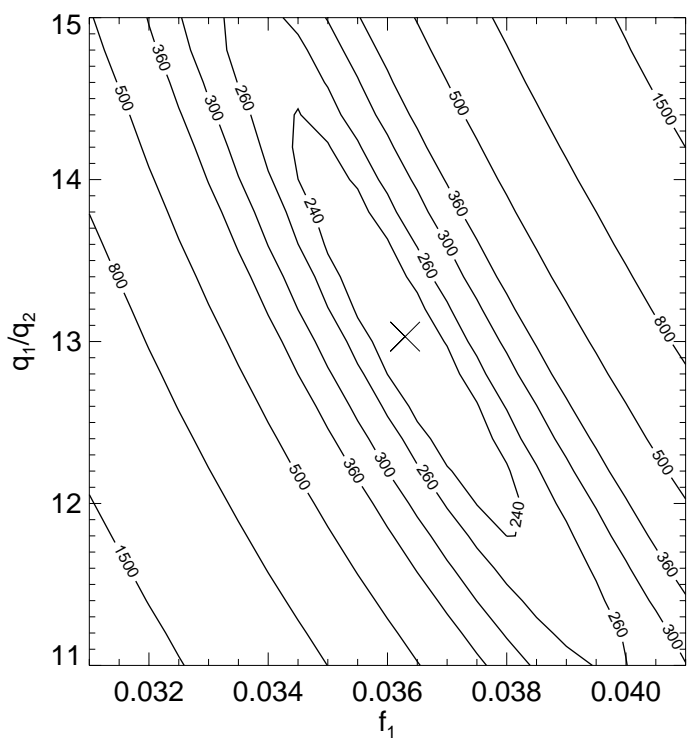

FIG. 4.-Contour of $\chi^{2}$ for parameters $f_{1}$ and $q_{1} / q_{2}$, fixing the emissivity laws $\left(\alpha_{1}, \alpha_{2}\right)$ to their best-fit values. Our best-fit two-component model is denoted by a cross.

thought of as a phenomenological "expansion set" for describing the composite dust spectrum.

\subsection{Fit Results for Specific Models}

We now describe our fits to eight different models of the form described above. All results from these fits are described in Table 1. In the first four we consider only a single component $\left(f_{1}=1\right)$ whose temperature varies on the sky. We strongly emphasize that none of our models force a constant temperature everywhere on the sky, which is an extremely poor description of the data (as can be seen in Figure $2 b$ ). For a given dust model, the temperature is uniquely constrained by the DIRBE $100 \mu \mathrm{m} / 240 \mu \mathrm{m}$ flux ratio along each line of sight. We perform single-component fits for $\alpha=1.5,1.7,2.0$, and 2.2, obtaining the best reduced $\chi_{v}^{2}$ at $\alpha=2.0$. This is in agreement with previous fits to the FIRAS data (Boulanger et al. 1996) and also with earlier theoretical prejudice (Draine \& Lee 1984).

These results are encouraging, but statistically a $\chi^{2}=3801$ for 123 degrees of freedom (for the $v^{2}$ model) is completely unacceptable. The first two-component model we consider is one designed to replicate the spectrum in Pollack et al. (1994). The prescription in their paper for their best-fit broken power law with $\left\langle T_{1}\right\rangle=\left\langle T_{2}\right\rangle$ corresponds in our model to $\alpha_{1}=1.5, \alpha_{2}=2.6, f_{1}=0.25$, and $q_{1} / q_{2}=0.61$. This results in a considerably better fit of $\chi_{v}^{2}=15.3$ without fitting any new free parameters. The choices for $\alpha_{1}, \alpha_{2}, f_{1}$, and $q_{1} / q_{2}$ are based upon other empirical evidence completely independent of the DIRBE and FIRAS data sets. The ratio of the Pollack et al. model to the straw-man $v^{2}$ model is shown in Figure $3 c$ as a light solid line. Between 800 and $1800 \mathrm{GHz}$, where the FIRAS signal is very good, the model matches the data to approximately $1 \%$ everywhere. At lower frequencies, the largest deviation is $25 \%$.

Allowing $f_{1}$ and $q_{1} / q_{2}$ to float with fixed $\alpha_{1}, \alpha_{2}$ provides even better fits. We attempt to reproduce the results found in Reach et al. (1995), where a component of very cold dust was proposed to explain the low-frequency excess. Reach et al. used $\alpha=2$ for both the warm and cold components as a mathematical convenience. Letting our model float with $\alpha_{1}=\alpha_{2}=2$ we obtain $f_{1}=0.00261$ and $q_{1} / q_{2}=2480$. This model achieves a better fit than any other model tested in the literature to date, yielding a $\chi^{2}=1241$, or $10.3 / \mathrm{DOF}$. A physical interpretation of this combination of parameters in the context of our models would imply that there is a component constituting $0.26 \%$ of the dust emission power, but with an opacity ratio 2480 times higher than the dominant component. This huge opacity ratio explains the low temperature, $\left\langle T_{1}\right\rangle=5 \mathrm{~K}$, as compared to the dominant component at $\left\langle T_{2}\right\rangle=18 \mathrm{~K}$. Since this compelling fit appeared in the Reach et al. (1995) paper, some authors have sought to explain the model in the context of this simple interpretation. Fractal grains (Fogel \& Leung 1998) and other possibilities have been raised to explain the opacity ratio, but no convincing mechanism has yet been proposed. Opacities may indeed differ considerably, but factors of many thousand are probably unreasonable. However, the idea of multiple well-mixed components at different temperatures deserves further exploration.

By taking the values from Pollack et al. (1994) of $\alpha_{1}=1.5$, $\alpha_{2}=2.6$, but realizing that such different components are very likely to be at different temperatures, we allow $f_{1}$ and $q_{1} / q_{2}$ to float, obtaining $\chi^{2}=244$ or $2.0 / \mathrm{DOF}$ for $f_{1}=$ 0.0309 and $q_{1} / q_{2}=11.2$. It may seem surprising at first that one component is 11 times "better" at thermally radiating than another, but to justify this we appeal to the empirical measurements of Agladze et al. (1996). They find that the amorphous silicate $\mathrm{MgO} \cdot 2 \mathrm{SiO}_{2}$ at $20 \mathrm{~K}$, for example, radiates $\sim 40$ times more readily at $300 \mathrm{GHz}$ than the crystalline silicate enstatite $\left(\mathrm{MgSiO}_{3}\right)$ (Agladze et al. 1996, Table 1). The effective optical opacities $\kappa^{*}$ may also vary significantly, so a wide range in emissivity ratios $q_{2} / q_{1}$ is empirically well established. Furthermore, our model requires only a tiny fraction of the dust to be of this kind. This is a very reasonable theoretical step to take to obtain a formal increase in likelihood of $\sim 350$ orders of magnitude over a simple $v^{2}$ model.

A further reduction to $\chi^{2}=219$ or $1.85 / \mathrm{DOF}$ is achieved by allowing the power-law indices $\alpha_{1}$ and $\alpha_{2}$ to vary. The best-fit values are $\alpha_{1}=1.67, \alpha_{2}=2.70, f_{1}=0.0363$, and $q_{1} / q_{2}=13.0$. A contour plot of $\chi^{2}$ as a function of $f_{1}$ and $q_{1} / q_{2}$ is shown in Figure 4 .

For these model parameters, the temperatures of the two components are related by

$$
T_{1}=0.352 T_{2}{ }^{1.18} \text {. }
$$

The mean temperatures are $\left\langle T_{1}\right\rangle=9.4 \mathrm{~K}$ and $\left\langle T_{2}\right\rangle=16.2$ $\mathrm{K}$ for the $71 \%$ of sky that we fit. This is the model we adopt for the comparisons discussed in the next section.

\section{DISCUSSION}

\subsection{Interpretation of Best-Fit Model}

The thermal emission from Galactic dust can be very successfully predicted at millimeter/microwave frequencies using a two-component composition model with temperature varying on the sky. We tentatively refer to the two components as an amorphous silicate-like component $\left(v^{1.7}\right.$ emissivity, $\langle T\rangle \approx 9.5 \mathrm{~K}$ ) and a carbonaceous component $\left(v^{2.7}\right.$ emissivity, $\left.\langle T\rangle \approx 16 \mathrm{~K}\right)$. This solution agrees with the FIRAS data much better than a two-component model using two $v^{2}$ emissivity components with one of the components very cold $(\langle T\rangle \approx 5 \mathrm{~K})$. Note also that we have obtained only four global model parameters from the entire 
FIRAS data set - all other column density and temperature information is derived from the DIRBE $100 \mu \mathrm{m}$ and $240 \mu \mathrm{m}$ maps. In the Reach et al. analysis, temperatures of the two components were allowed to float independently and were fitted directly to the FIRAS data.

Although our analysis does nothing to rule it out, we find no evidence for a recently proposed warm component $\left(v^{1}\right.$ emissivity, $\langle T\rangle \approx 29 \mathrm{~K}$ ) associated with the WIM. This component results from a different approach to modeling the dust emission spectrum (see Lagache et al. 1999 for details).

\subsection{Spatial Coherence of Dust Properties}

Previous two-component models of dust emissivity fitted to the FIRAS data found that the two components must be spatially correlated to a high degree (Reach et al. 1995). We demonstrate this fact by computing the correlation slope of dust with FIRAS $500 \mathrm{GHz}$ as shown in Figure 2. Removing this correlated emission reduces the variance in the 500 $\mathrm{GHz}$ map by $95 \%$. Based on the data at high Galactic latitude, about $4 \%$ of the variance is attributable to measurement noise. This leaves $1 \%$ of the variance (or $10 \%$ of the signal) as the upper limit for uncorrelated $500 \mathrm{GHz}$ emission. If there exists a separate cold dust component which does not emit at $100 \mu \mathrm{m}$, then it must be very highly spatially correlated with warm dust.

\subsection{Evidence for Variations in Dust Properties}

The agreement between the best-fit two-component model and the FIRAS data is impressive (see Figs. 5 and 6). The reduced $\chi_{v}^{2}$ is close to unity, implying that the model uncertainties are small compared to the measurement errors. The far-IR/millimeter sky at $|b| \gtrsim 15^{\circ}$ appears to be well fitted by a fixed model for the interstellar dust. The temperature varies, but the composition and size distribution of the dust grains are constrained to be very similar everywhere in the diffuse ISM.

Although our best-fit model appears to describe successfully the average dust emission spectrum, there still might be systematic variations across the sky. Splitting the sky into different zones based upon various observables, one can search for regions that deviate from our model. Dividing the sky according to temperature, we do not find significant differences (see Fig. 5).

However, splitting the sky according to dust/gas ratio, one does find coherent differences. We construct a dust/gas ratio by dividing the SFD98 dust map by the LeidenDwingeloo H I map (Hartmann \& Burton 1997), both smoothed to a $45^{\prime}$ FWHM Gaussian beam. The SFD98 dust map is proportional to the $100 \mu \mathrm{m}$ emission expected if the entire sky were at a uniform temperature. Regions where the dust/gas ratio exceeds the high-latitude average by more than a factor of 2 are designated "molecular" $(14 \%$ of the sky). Remaining pixels are designated "atomic" $(40 \%)$. The remaining $46 \%$ of the sky is excluded by our FIRAS mask or by the lack of Leiden-Dwingeloo survey data at $\delta<-30^{\circ}$.

In Figure 6, we plot the correlation slope between our model predictions and the FIRAS data for the "molecular" and "atomic" sky. Since the full-sky fits are dominated by the "molecular" sky, the model very nearly fits that zone with a correlation slope near unity at all frequencies (Fig. $6 b$ ). However, "the atomic" sky shows deviations relative to the model that approach $\sim 15 \%$ at low frequencies (Fig. $6 c$ ).
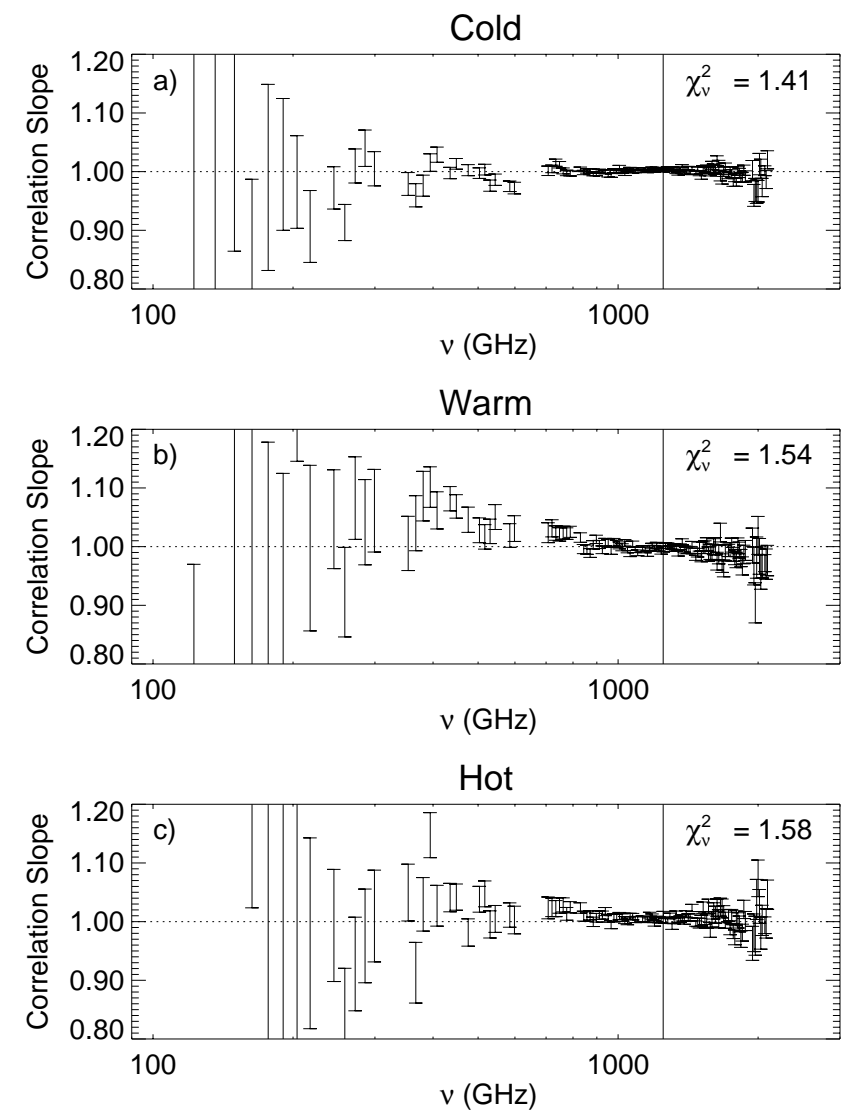

FIG. 5.-FIRAS vs. best-fit model correlation slopes. The sky is divided into three zones based upon temperature: (a) cold regions $(\mathscr{R}=$ $\left.I_{100 \mu \mathrm{m}} / I_{240 \mu \mathrm{m}}<0.62\right)$, (b) warm regions $(0.62<\mathscr{R}<0.69)$, and $(c)$ hot regions $(\mathscr{R}>0.69)$. The systematic residuals between zones is not more than $\sim 5 \%$. The vertical line is drawn at $240 \mu \mathrm{m}$, where the models are constrained to fit the DIRBE measurements.

We suspect that these differences between "atomic" and "molecular" zones represent true variations in dust grain properties. These variations can be quantified by adjusting our model parameters to achieve a better fit in the "atomic" zone. The fits can be improved by retaining the best-fit parameters in the molecular zone and adjusting $\alpha_{1}$ lower, $f_{1}$ higher, or $q_{1} / q_{2}$ higher in the atomic zone. Lowering $\alpha_{1}$ does not improve the fit as well and would require a qualitative change in the millimeter opacities of the "silicate" component. We consider this possibility the least likely, though it is possible that we are seeing ice mantle accretion or some other environment-dependent mechanism.

In this region of parameter space, $f_{1}$ and $q_{1} / q_{2}$ are sufficiently degenerate that an almost equally good fit may be obtained in the atomic zone by adjusting either parameter a modest amount (Fig. 4). Either $f_{1}$ can be increased by $\sim 15 \%$ or $q_{1} / q_{2}$ increased by $\sim 25 \%$ in the atomic zone (see Fig. $6 c$ ). Increasing $f_{1}$ is equivalent to increasing the amount of the "silicate" component relative to the warmer "carbon-based" component. The change in $q_{1} / q_{2}$ could very plausibly be interpreted as a decrease in $\kappa_{2}^{*}$ (an increase in $q_{2}$ ) in the molecular zone. If the second component is physically composed of carbon-based grains, it might be responsible for the UV absorption bump at $2175 \AA$ and therefore be sensitive to saturation of that feature. Much of the molecular zone has an SFD98 extinction $A(V) \gtrsim 0.5$ 

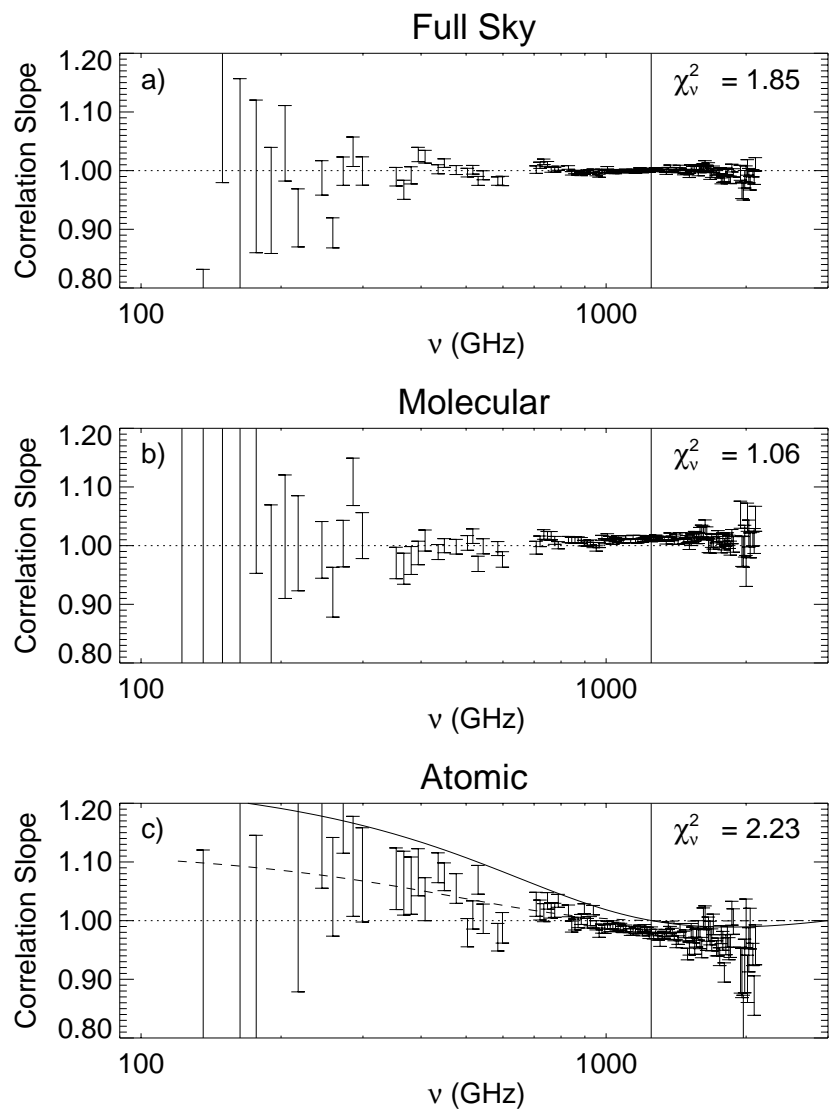

FIG. 6.-FIRAS vs. best-fit model correlation slopes. The sky is divided into two zones based upon dust/gas ratio: (a) both atomic- and moleculardominated zones, $(b)$ zones dominated by molecular clouds, and $(c)$ zones dominated by atomic gas. The maximum deviation is $\sim 15 \%$ at $100 \mathrm{GHz}$ for the atomic zone. For the atomic gas, we overplot our best-fit model modified to $f_{1}=0.0465$ (solid line) or modified to $q_{1} / q_{2}=15.0$ (dashed line). The vertical line is drawn at $240 \mu \mathrm{m}$, where the models are constrained to fit the DIRBE measurements.

mag, which corresponds to $A(2175 \AA) \gtrsim 1$ mag. When this feature begins to saturate, the carbon grains see a change in the ISRF that effectively reduces $\kappa_{2}^{*}$. The small differences we see between the "atomic" and "molecular" zones are certainly consistent with spatial variations in the radiation field, which we consider to be one of the most reasonable explanations. Note that we have not proved that this mechanism is responsible, or even that the two dominant components are silicate and carbon-dominated grains. However the data are consistent with this interpretation.

\section{APPLICATION TO CMBR ANISOTROPY}

We compare predictions from our best-fit twocomponent model to the COBE DMR data. Significant microwave emission from dust was found by Kogut et al. (1996) by using DIRBE $140 \mu \mathrm{m}$ flux as a dust template. As a similar comparison, we compute the correlation slope in each DMR channel with respect to our model. This slope is sensitive only to emission correlated with the dust and does not depend on isotropic backgrounds. For the purposes of these fits, DMR pixel $i$ is weighted by the inverse of $\sigma_{i}^{2}$ $+\sigma_{\mathrm{CMB}}^{2}$, where $\sigma_{i}$ is the measurement noise in pixel $i$ and $\sigma_{\mathrm{CMB}}$ is the rms power in the CMB anisotropy, taken to be $30 \mu \mathrm{K}$ (Bennett et al. 1996).

The first column in Table 3 is the correlation slope of DMR against our best-fit model evaluated at $500 \mathrm{GHz}$. This frequency is chosen because it is low enough to be on the Rayleigh-Jeans side of the Planck function, so the dust spectral index between DMR and our $500 \mathrm{GHz}$ predictions has very little temperature dependence. Also, this frequency is high enough that FIRAS obtained high quality data for the dust emission. As Figure 2 has demonstrated, our model is well tested at $500 \mathrm{GHz}$ and one may be confident that it represents real dust emission on the sky. Therefore, 500 $\mathrm{GHz}$ is a sensible reference frequency to use in such comparisons. The next column of the table is the rms power in $\mu \mathrm{K}$ brightness temperature implied by this correlation slope. Note that we are confined to the mask used in the computation of the DMR CMBR anisotropy. A less exclusive mask would yield a higher rms power. The remaining columns of Table 3 are similar but use the $140 \mu \mathrm{m}$ DIRBE map instead of our prediction, for direct comparison with the Kogut et al. results. However, as we have already seen in Figure 2, the correlation at $500 \mathrm{GHz}$ is not tight and is probably worse at DMR frequencies. These comparisons in Table 3 are meant to indicate the spectral shape of dustcorrelated microwave emission.

It is clear in Table 3 that the dust-correlated emission at $31 \mathrm{GHz}$ is larger than at $53 \mathrm{GHz}$ - when according to our model it should have fallen by a factor of $\sim 5$. Comparison of DMR data with our model evaluated at the same frequency gives an idea of the amount of the excess. Again, correlation slopes are computed, for DMR data as a function of model predictions, with a slope of unity corresponding to an accurate model. The results are tabulated in Table 4. At $90 \mathrm{GHz}$, there is $20 \%$ more dust-correlated emission than predicted. At 53 and $31.5 \mathrm{GHz}$, this emission is too high by a factor of 2.4 and 20, respectively. Similar excess emission is seen at 14.5 and $31 \mathrm{GHz}$ from the data of Leitch et al. (1997) and at similar frequencies in the Saskatoon data

TABLE 3

COMPaRISON OF DUST TeMPlates WiTH DMR

\begin{tabular}{ccccr}
\hline \hline $\begin{array}{c}v \\
(\mathrm{GHz})\end{array}$ & $\begin{array}{c}\text { Model}(500 \mathrm{GHz}) \\
\text { Slope } \times 10^{-3}\end{array}$ & \multicolumn{1}{c}{$\begin{array}{c}\mathrm{rms} \\
(\mu \mathrm{K})\end{array}$} & $\begin{array}{c}\text { DMR } / 140 \mu \mathrm{m} \\
\text { Slope } \times 10^{-4}\end{array}$ & \multicolumn{1}{c}{$\begin{array}{c}\mathrm{rms} \\
(\mu \mathrm{K})\end{array}$} \\
\hline $31.5 \ldots \ldots$ & $1.81 \pm 0.27$ & $20.5 \pm 3.1$ & $1.81 \pm 0.28$ & $20.6 \pm 3.2$ \\
$53 \ldots \ldots \ldots$ & $1.35 \pm 0.29$ & $5.4 \pm 1.1$ & $0.99 \pm 0.29$ & $4.0 \pm 1.2$ \\
$90 \ldots \ldots \ldots$ & $4.27 \pm 1.08$ & $5.9 \pm 1.5$ & $3.38 \pm 1.10$ & $4.7 \pm 1.5$ \\
\hline
\end{tabular}

NoTE.-Correlation slopes of the DMR channels with best-fit $500 \mathrm{GHz}$ prediction and $140 \mu \mathrm{m}$ DIRBE data. Slope values are dimensionless flux ratios. The rms values are the rms power, in $\mu \mathrm{K}$, expected in the DMR maps due to the dust emission traced by template. These values compare to an rms power of $29 \pm 1 \mu \mathrm{K}$ due to CMBR anisotropy. 
TABLE 4

EXCESS DUST-CORRELATED DMR EMISSION

\begin{tabular}{cccc}
\hline \hline $\begin{array}{c}v \\
(\mathrm{GHz}) \\
(1)\end{array}$ & $\begin{array}{c}\text { DMR/Model }(v) \\
(2)\end{array}$ & $\begin{array}{c}\text { Percent Variance } \\
(3)\end{array}$ & $\begin{array}{c}\text { rms Power } \\
(\mu \mathrm{K}) \\
(4)\end{array}$ \\
\hline $31.5 \ldots \ldots$ & $20.09 \pm 3.09$ & 0.87 & $20.2 \pm 3.1$ \\
$53 \ldots \ldots \ldots$ & $2.45 \pm 0.50$ & 0.57 & $5.6 \pm 1.1$ \\
$90 \ldots \ldots \ldots$ & $1.18 \pm 0.29$ & 0.42 & $6.2 \pm 1.5$ \\
\hline
\end{tabular}

NoTE.-Excess dust-correlated microwave emission measured by DMR. Col. (1): Frequency of DMR channel, in GHz. Col. (2): Correlation slope of DMR emission vs. model predictions. Col. (3): Percent of variance in DMR data accounted for by this dust. Note that the vast majority of the variance is receiver noise. Col. (4): rms power due to dust emission, in $\mu \mathrm{K}$ brightness temperature.

(Netterfield et al. 1995; Netterfield et al. 1997; de OliveiraCosta et al. 1997).

This excess microwave emission is clearly correlated with the dust but is not due to its thermal (vibrational) emission. It has recently been suggested that magnetic dipole emission from paramagnetic grains (Draine \& Lazarian 1999) or electric dipole emission from rapidly spinning dust grains (Draine \& Lazarian 1998b) could dominate at these frequencies. Others have suggested that dust-correlated freefree emission may be responsible (de Oliveira-Costa et al. 1998). However, Draine and Lazarian argue against this on energetic grounds in Draine \& Lazarian (1998a). Galactic synchrotron emission is not a favored explanation because it is unlikely to be highly correlated with the dust.

\subsection{Templates for CMBR Contamination}

It is critical that CMBR experimentalists compare their observations with valid models for the Galactic dust emission. A "template approach" is often carelessly used to compare observations with expected contaminants, with the correlation amplitude indicating the level of contamination. For example, $100 \mu \mathrm{m}$ emission maps (e.g., IR $A S$ or DIRBE) or $21 \mathrm{~cm}$ maps (e.g., Leiden-Dwingeloo: Hartmann \& Burton 1997) are often used as templates for microwave dust emission. These templates ignore well-measured variations in dust temperature and variations in the dust/gas ratio. We demonstrate this point by differencing the broadband FIRAS $500 \mathrm{GHz}$ map (Fig. 7) with best-fit templates. The residuals with respect to the $\mathrm{H}$ I template (Fig. 8) or the $100 \mu \mathrm{m}$ template (Fig. 9) are noticeably worse than with our model prediction (Fig. 10). In addition, the $\mathrm{H}$ I or $100 \mu \mathrm{m}$ template offers no insight as to frequency dependence of the dust emission. Because the $100 \mu \mathrm{m}$ template is so sensitive to the temperature, it may be the worst of these at high Galactic latitude.

This need to use the proper template will grow with future data sets. In the case of the DMR data, no adverse effects resulted from the use of the $140 \mu \mathrm{m}$ template, as can be seen in Table 3. The expected rms power from dust in the DMR channels is not significantly altered by using our model. However, the signal-to-noise ratio of DMR is much less than 1 per pixel. The measurement noise overwhelms the template errors in this case. In the case of $\mathrm{S} / \mathrm{N} \sim 1$ data, e.g., FIRAS $500 \mathrm{GHz}$, it is apparent from Figure 2 that our template is more accurate, and much more readily detected,

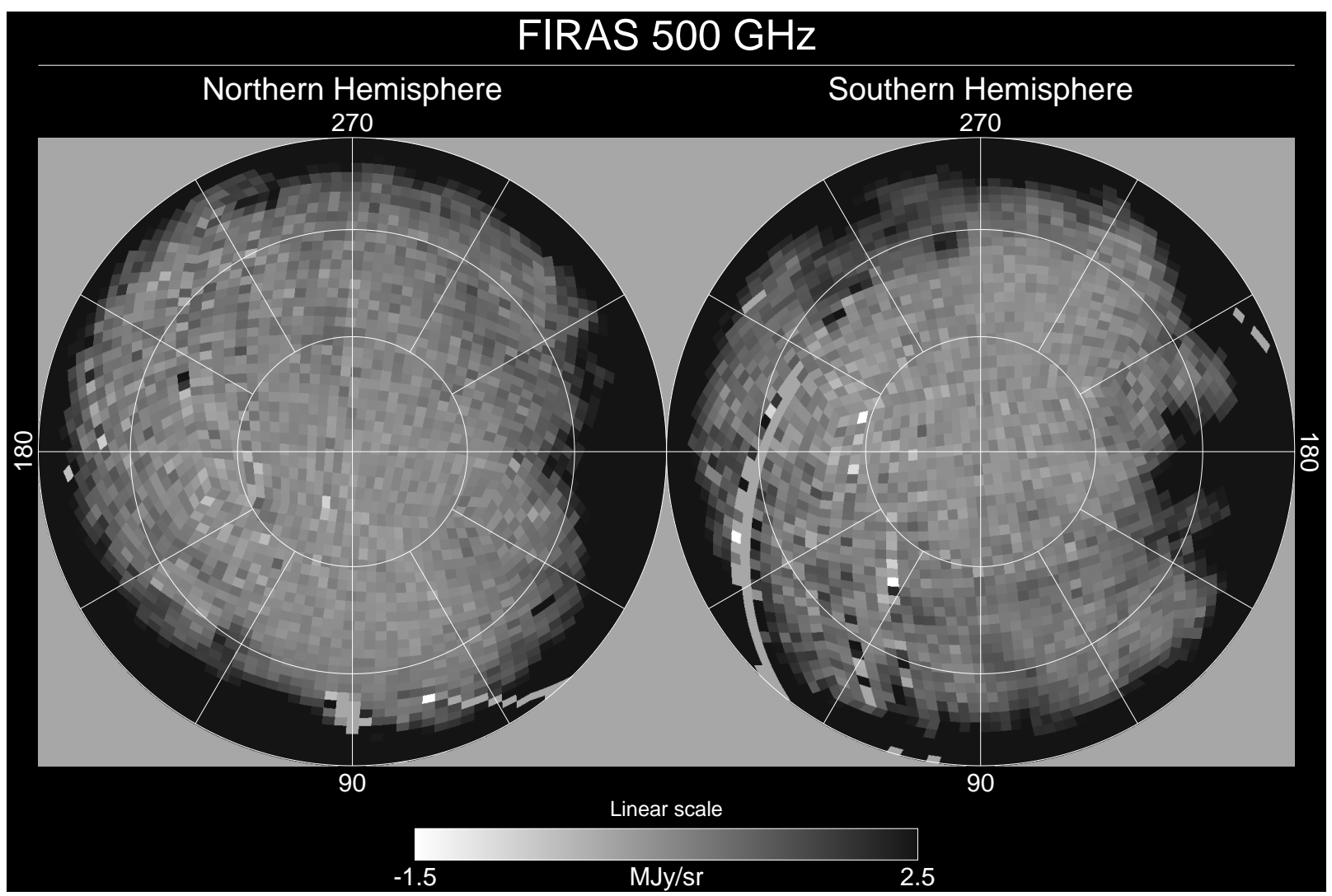

FIG. 7.-FIRAS broadband $500 \mathrm{GHz}$ map, as defined in the text (eq. [3]). These Lambert ZEA polar projections are centered on the NGP (left), and SGP (right), with Galactic longitude labeled in degrees. Lines of constant latitude and longitude are spaced every $30^{\circ}$. 


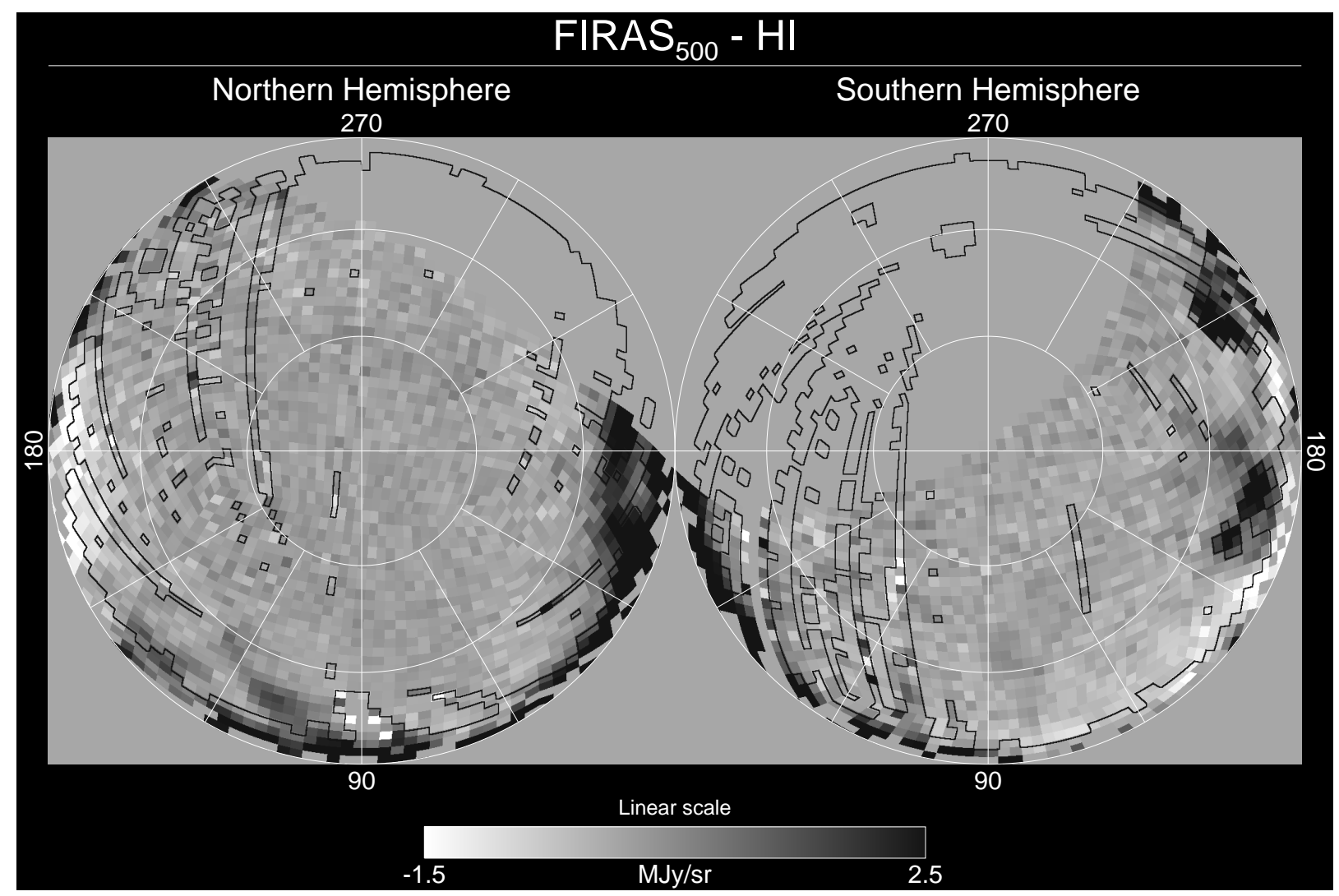

FIG. 8.-Difference map between the broadband FIRAS $500 \mathrm{GHz}$ map and the best-fit $\mathrm{H}$ I template convolved to the same beam shape. Declinations south of $\delta=-30^{\circ}$ were not observed in the Leiden-Dwingeloo (Hartmann \& Burton 1997) survey, accounting for the blank regions of missing data. The thin black lines outline FIRAS pixels masked from our analysis (see $§ 2.4 .2$ ).

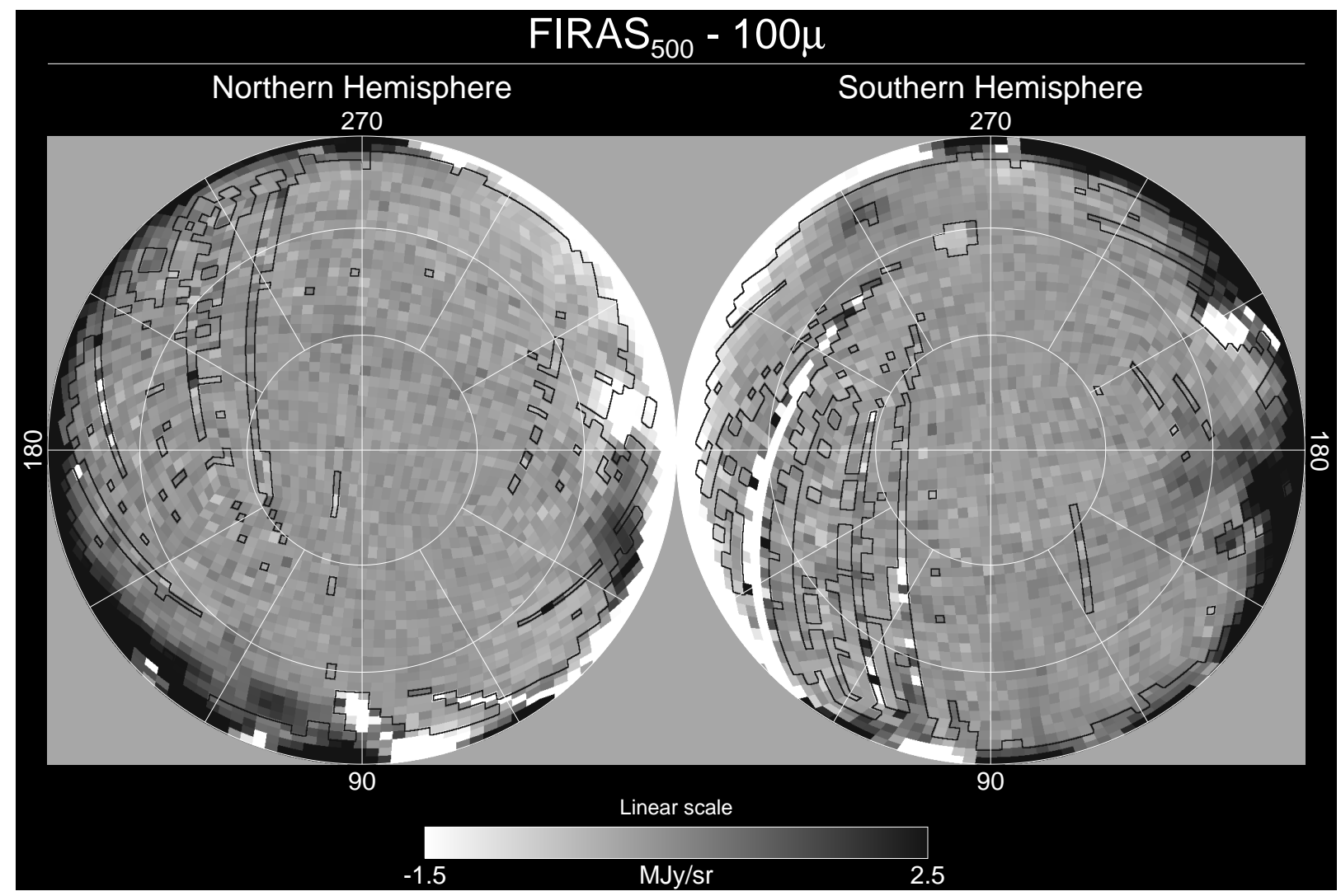

FIG. 9.-Difference map between the broadband FIRAS $500 \mathrm{GHz}$ map and a best-fit $100 \mu \mathrm{m}$ template convolved to the same beam shape 


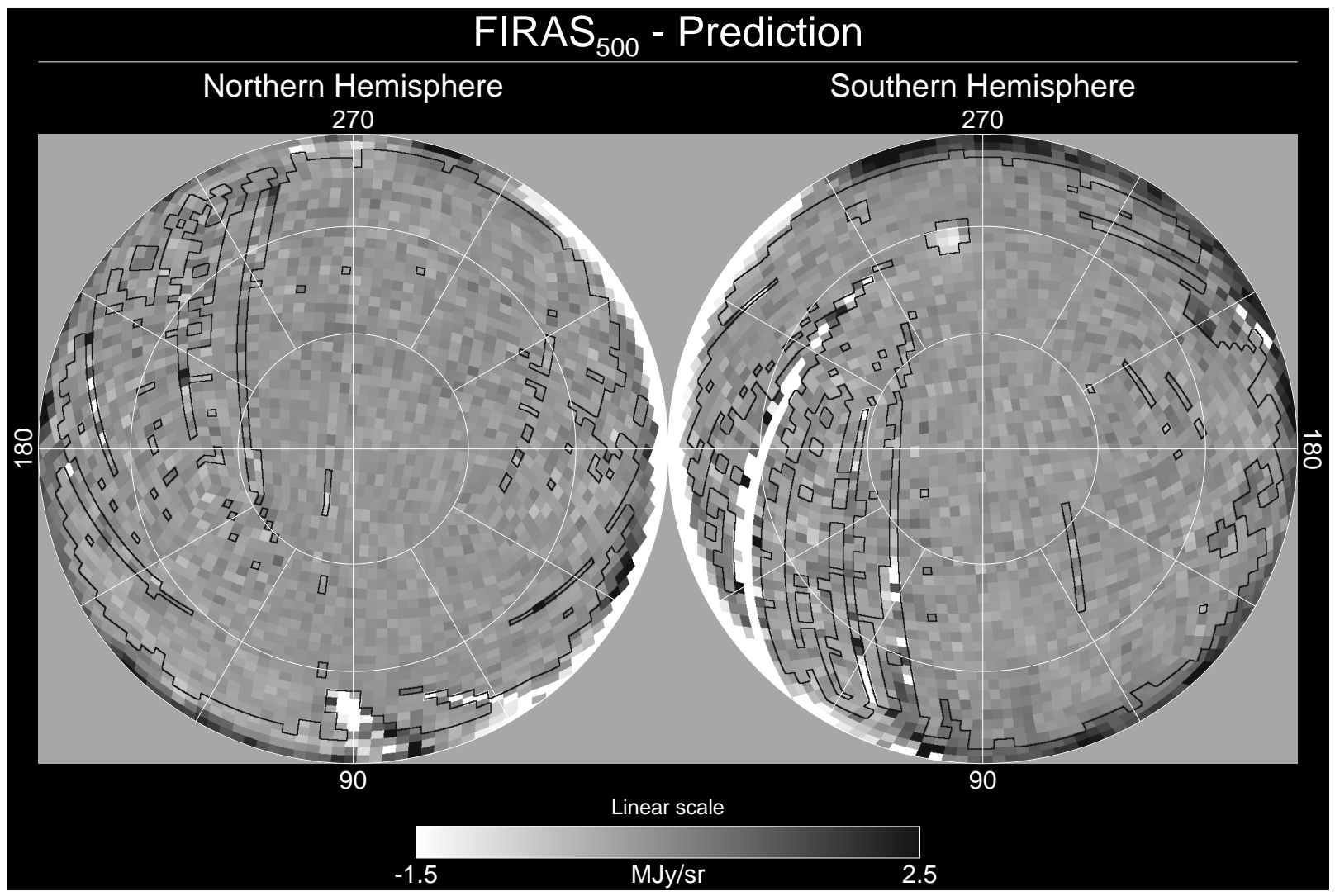

FIG. 10.-Difference map between the broadband FIRAS $500 \mathrm{GHz}$ map and the emission predicted from our best-fit two-component dust model convolved to the same beam shape. The residuals are noticeably smaller than residuals obtained by using either an $\mathrm{H}$ I template (Fig. 8) or $100 \mu \mathrm{m}$ template (Fig. 9).

than $100 \mu \mathrm{m}$ flux or H I column. These considerations will be even more important with satellites such as $M A P\left(13^{\prime}\right)$ because our map is the only full-sky well calibrated dust model at high resolution $\left(6^{\prime}\right)$.

We would encourage CMBR researchers to present measurements of dust-correlated microwave emission by using our predictions as a baseline, so that (at least where vibrational dust emission dominates) the comparison is free of temperature-dependent biases and assumptions about the dust/H I ratio. This will allow easy comparison of samples from various parts of the sky - a comparison that is quite difficult with current dust templates.

\section{SUMMARY AND CONCLUSIONS}

We have demonstrated that the SFD98 $100 \mu \mathrm{m}$ emission map, extrapolated with a very simple two-component dust model, is an excellent predictor of the Galactic emission as seen by FIRAS at all frequencies. Although the older SFD98 $v^{2}$ emission predictions are tightly correlated with the FIRAS data, the correlation slope deviates significantly from unity at frequencies far from $1250 \mathrm{GHz}$ and $3000 \mathrm{GHz}$ ( 240 and $100 \mu \mathrm{m}$ ) where it is constrained by the DIRBE data. The $v^{2}$ emissivity assumed by SFD 98 produces a reduced $\chi_{v}^{2} \approx 30$ when compared with the FIRAS data. Although this fit is unacceptable, no other singlecomponent power law emissivity model improves $\chi_{v}^{2}$ significantly.

We provide a general multicomponent model in which each component is described by an emissivity power law, $\alpha$, power fraction, $f$, and a ratio of thermal emission to optical opacity, $q$. Each component is required to be in equilibrium with the ISRF. This couples the temperatures of each component and uniquely constrains them along each line of sight by the DIRBE $100 / 240 \mu \mathrm{m}$ ratio.

In addition to one-component models evaluated for various emissivity power law indices, we evaluate four twocomponent models. They correspond to Pollack et al., Reach et al., our best fit using Pollack et al. emissivities, and our-best overall fit for all four model parameters. Our bestfit parameters are physically reasonable, given empirical evidence found in Agladze et al. (1996). See Table 1 for a summary of our results.

The data argue very strongly that the dust properties of the ISM are uniform over virtually the entire high-latitude sky on angular scales greater than $7^{\circ}$. We have found marginal evidence for variations in the molecular-dominated zones relative to atomic-dominated zones. We tentatively suggest that these variations are due to UV optical depth effects within the molecular zones.

This thermal (vibrational) dust emission model fails to explain dust-correlated microwave emission observed by DMR. The $90 \mathrm{GHz}$ emission is in approximate agreement with our model, but the 53 and $31 \mathrm{GHz}$ DMR emission is high by factors of 2.4 and 20, respectively. This excess emission could result from rapidly spinning dust grains (Draine \& Lazarian 1998b) or from free-free emission. Whatever the emission mechanism, it must be strongly correlated with the thermal (vibrational) dust emission. 
Predictions of our best-fit model for thermal dust emission are available on the World Wide Web. ${ }^{3}$ A description of the data products and a list of caveats are provided in Appendix C.

Conversations with Dale Fixsen contributed enormously to our understanding of the FIRAS data. We would also like to thank Bruce Draine, Carl Heiles, Dave Hollenbach,

\footnotetext{
${ }^{3}$ The data are publicly available via the World Wide Web at http:// astro.berkeley.edu/dust and links therein.
}

Chris McKee, David Spergel, Eric Gawiser, and Jeffrey Newman for encouragement and helpful discussions. An anonymous referee helped clarify a number of points. Computers were partially provided by a Sun AEGP Grant. D. P. F. is an NSF Graduate Fellow. D. J. S. is partially supported by the MAP project and by the Sloan Digital Sky Survey. This work was supported in part by NASA grants NAG 5-1360 and NAG 5-7833. The COBE data sets were developed by the NASA Goddard Space Flight Center under the guidance of the COBE Science Working Group and were provided by the NSSDC.

\section{APPENDIX A}

\section{FURTHER PROCESSING OF THE FIRAS DATA}

The FIRAS Pass 4 data products are released in two sets of frequency bins, corresponding to the two sides of the instrument. There are 43 bins in the low-frequency (LOWF) set, running from 68.02 to $639.37 \mathrm{GHz}$, and 170 bins in the high-frequency (HIGH) set, running from 612.19 to $2911.29 \mathrm{GHz}$. The bin spacing is $13.6041 \mathrm{GHz}$, giving a 3 bin overlap between LOWF and HIGH.

\section{A1. RECALIBRATION}

An error in the FIRAS external calibrators is described in Mather et al. (1999). The thermometers were found to be miscalibrated by $5 \mathrm{mK}$, causing a systematic error in LOWF. It is expected that this miscalibration introduces an error of less than $1 \%$ in the LOWF data, which is negligible for the purposes of this paper. Therefore we have chosen to ignore this problem in the data.

Comparisons of the high-frequency FIRAS data to the DIRBE $240 \mu \mathrm{m}$ data shows an inconsistency at the $1 \%$ level. The DIRBE gain is uncertain at this level owing to the uncertainties in its filter response and calibration technique. However, we have chosen to reduce all the FIRAS measurements by $1 \%$, which is well within the gain uncertainty of the HIGH data, and within the measurement noise of the LOWF data. Because the covariance of neighboring FIRAS frequency bins is embodied in the FIRAS covariance matrix, our results are only weakly dependent upon this $1 \%$ recalibration.

\section{A2. BAD BINS}

Line emission from $\mathrm{CO},\left[\mathrm{C}_{\mathrm{I}}\right],[\mathrm{N}$ II], [C II], [O I ] , and $\mathrm{CH}$ was detected. We have excluded those bins and bins corresponding to $\mathrm{O}_{2}, \mathrm{H}_{2} \mathrm{O}$, and [Si I], even though no emission was detected by the FIRAS team. A few bins were excluded because of residuals in the mirror transport mechanism (MTM) ghost removal. Several bins are excluded from our analysis in the frequency range 639.37 to $680.21 \mathrm{GHz}$ for three reasons: (1) inefficiencies in the dichroic splitter, (2) a destructive interference pattern caused by reflection off of the plastic holder of one of the optical elements, and (3) an aliased MTM sideband. All of these effects taken together overwhelm the signal in these bins and justify their exclusion. A summary of the frequency bins excluded in our analyses is found in Table 5 .

Our analyses make use of 123 frequency bins at $100<v<2100 \mathrm{GHz}$. At lower frequencies, the $\mathrm{S} / \mathrm{N}$ of the dust emission is less than 1. At higher frequencies, the absolute error in the FIRAS gain exceeds $2 \%$ due to uncertainties in the bolometer calibration (see FIRAS Exp. Supp. 1997, § 7.3.2).

\section{APPENDIX B}

\section{COMPUTATIONAL METHODS}

Comparison between the Galactic dust emission as observed by FIRAS and our predictions is computationally challenging. The predictions are made at the resolution of DIRBE to take full advantage of temperature information on scales of $\sim 1^{\circ}$. These DIRBE-resolution predictions are then smoothed to the FIRAS beam and compared to 4376 FIRAS pixels (71\% of the sky) at 123 frequencies, for a total of $\sim 540,000$ data value comparisons.

\section{B1. COMPUTATION OF SPECTRAL SHAPE}

Aside from the four global parameters $\left(f_{1}, q_{1} / q_{2}, \alpha_{1}, \alpha_{2}\right)$, the model predictions are made only from the DIRBE observations at 100 and $240 \mu \mathrm{m}$. We make use of the DIRBE $100 \mu \mathrm{m}$ map described in SFD98 with zodiacal light and the cosmic infrared background removed. Our dust temperatures are always derived from a ratio map, $\mathscr{R}$, which is a filtered $I_{100} / I_{240}$ 
TABLE 5

FIRAS BAD FREQUENCY BINS

\begin{tabular}{|c|c|c|c|c|}
\hline Reason & Channel & $\begin{array}{c}v \\
\left(\mathrm{~cm}^{-1}\right)\end{array}$ & $\begin{array}{c}v \\
(\mathrm{GHz})\end{array}$ & $\begin{array}{c}\lambda \\
(\mu \mathrm{m})\end{array}$ \\
\hline $\operatorname{CO}(J=1-0) \ldots \ldots$ & 3 & 3.631 & 108.83 & 2753.8 \\
\hline $\mathrm{CO}(J=2-1) \ldots \ldots$ & 12 & 7.717 & 231.27 & 1295.9 \\
\hline МTM ............... & 18 & 10.440 & 312.90 & 957.8 \\
\hline MTM ............... & 19 & 10.894 & 326.50 & 917.9 \\
\hline $\mathrm{CO}(J=3-2) \ldots \ldots$. & 20 & 11.348 & 340.10 & 881.2 \\
\hline $\mathrm{O}_{2} \ldots \ldots \ldots \ldots \ldots \ldots$ & 26 & 14.072 & 421.73 & 710.6 \\
\hline $\mathrm{CO}(J=4-3) \ldots \ldots$. & 29 & 15.433 & 462.54 & 647.9 \\
\hline$\left[\mathrm{C}_{\mathrm{I}}\right] \ldots \ldots \ldots \ldots \ldots$ & 31 & 16.341 & 489.75 & 611.9 \\
\hline $\mathrm{H}_{2} \mathrm{O} \ldots \ldots \ldots \ldots \ldots$ & 36 & 18.611 & 557.77 & 537.3 \\
\hline $\mathrm{CO}(J=5-4) \ldots \ldots$. & 37 & 19.065 & 571.37 & 524.5 \\
\hline Dichroic ............. & 40 & 20.427 & 612.19 & 489.6 \\
\hline Dichroic ............. & 41 & 20.881 & 625.79 & 478.9 \\
\hline Dichroic ....... & 42 & 21.335 & 639.40 & 468.7 \\
\hline Dichroic .... & $43-48$ & 21.6 & 646 & 464 \\
\hline $\mathrm{CO}(J=6-5)$ & 49 & 23.150 & 693.81 & 432.0 \\
\hline $\mathrm{CO}(J=7-6) \ldots \ldots$. & 57 & 26.782 & 802.65 & 373.4 \\
\hline$\left[\mathrm{C}_{\mathrm{I}}\right] \ldots \ldots \ldots \ldots \ldots$ & 58 & 27.236 & 816.25 & 367.2 \\
\hline $\mathrm{H}_{2} \mathrm{O} \ldots \ldots \ldots \ldots \ldots$ & 80 & 37.222 & 1115.54 & 268.7 \\
\hline$[\mathrm{N}$ II $] \ldots \ldots \ldots \ldots \ldots$ & 105 & 48.570 & 1455.65 & 205.9 \\
\hline $\mathrm{H}_{2} \mathrm{O}(J=2-1) \ldots \ldots$ & 124 & 57.195 & 1714.12 & 174.8 \\
\hline$[\mathrm{C}$ II $] \ldots \ldots \ldots \ldots \ldots$ & 137 & 63.096 & 1890.98 & 158.5 \\
\hline$[\mathrm{C}$ II $] \ldots \ldots \ldots \ldots \ldots$ & 138 & 63.550 & 1904.58 & 157.4 \\
\hline$[\mathrm{C}$ II $] \ldots \ldots \ldots \ldots \ldots$ & 139 & 64.004 & 1918.19 & 156.2 \\
\hline$[\mathrm{C}$ II $] \ldots \ldots \ldots \ldots \ldots$ & 140 & 64.457 & 1931.79 & 155.1 \\
\hline$\left[\mathrm{O}_{\mathrm{I}}\right] \ldots \ldots \ldots \ldots \ldots$ & 149 & 68.543 & 2054.23 & 145.9 \\
\hline$[\mathrm{Si} \mathrm{I}] \ldots \ldots \ldots$ & 168 & 77.167 & 2312.71 & 129.6 \\
\hline$[\mathrm{N}$ II $] \ldots \ldots \ldots \ldots \ldots$ & 179 & 82.161 & 2462.35 & 121.7 \\
\hline $\mathrm{CH}(J=2-1) \ldots \ldots$. & 188 & 86.246 & 2584.79 & 115.9 \\
\hline
\end{tabular}

NoTE.-The excluded FIRAS bins are listed, along with the reason for their exclusion. The LOWF and HIGH data overlap, with bins 40-42 covering the same frequencies as bins 43-45. Bins removed because of mirror transport mechanism ghosts are labeled "MTM," and those contaminated by the dichroic beam splitter are labeled "Dichroic."

flux ratio in the DIRBE passbands. For a multicomponent dust model, this ratio map measures the following combination of model parameters:

$$
\mathscr{R}_{p}=\frac{\sum_{k} K_{100}\left(\alpha_{k}, T_{p k}\right) I_{100}\left(T_{p k}\right)}{\sum_{k} K_{240}\left(\alpha_{k}, T_{p k}\right) I_{240}\left(T_{p k}\right)} .
$$

For more than one component, the temperatures are related via equation (14). For each model, we tabulate $\mathscr{R}$ as a function of the warmer component, $T_{2}$, as described by equations (14) and (B1). We fit a sixth-order polynomial to the curve $\ln T_{2}(\ln \mathscr{R})$ for the domain $10<T_{2}<31 \mathrm{~K}$. For our best-fit two-component model,

$$
\begin{aligned}
\ln T_{2}= & 2.872+0.2407 \ln \mathscr{R}+2.962 \times 10^{-2} \ln ^{2} \mathscr{R}+4.719 \times 10^{-3} \ln ^{3} \mathscr{R} \\
& +9.320 \times 10^{-4} \ln ^{4} \mathscr{R}+1.109 \times 10^{-4} \ln ^{5} \mathscr{R} .
\end{aligned}
$$

At each DIRBE pixel $p$, we read the values of the $100 \mu \mathrm{m}$ flux and the ratio $\mathscr{R}$, which in turn recovers $T_{1}$ and $T_{2}$. The flux at any frequency is then given by equation (1) for a one-component model or equation (6) for models with more than one component.

Conceptually, the DIRBE-based predictions are convolved with the FIRAS beam before comparison to the FIRAS data. In practice, such a straightforward approach proved too computationally expensive when minimizing the residuals over several model parameters. The dust temperature variations are rarely large within one FIRAS beam, allowing us to make approximations for the temperature distribution within a beam. Let $i$ index the 6144 FIRAS pixels and $j$ index the DIRBE map pixels. Within each FIRAS beam centered on FIRAS pixel $i$, our predictions can be explicitly expressed as

$$
F_{i}(v)=\sum_{j} B_{i j} I_{j} Y\left(v, \mathscr{R}_{j}\right),
$$


where $B_{i j}$ describes the beam pattern (the fractional contribution of pixel $j$ to FIRAS pixel $i$ ), $I_{j}$ is the $100 \mu \mathrm{m}$ flux in DIRBE pixel $j$, and $Y\left(v, \mathscr{R}_{j}\right)$ describes the model spectral shape,

$$
Y\left(v, \mathscr{R}_{j}\right)=\frac{I_{j}\left(v, \mathscr{R}_{j}\right)}{I_{j}\left(v_{0}, \mathscr{R}_{j}\right)} .
$$

The beam pattern is normalized to unity,

$$
\sum_{j} B_{i j}=1
$$

for each FIRAS pixel. Equation (B3) can be rewritten as

$$
F_{i}(v)=\left\langle I_{i}\right\rangle \sum_{j} W_{i j} Y\left(v, \mathscr{R}_{j}\right),
$$

where we have defined a weighted mean for the $100 \mu \mathrm{m}$ flux,

$$
\left\langle I_{i}\right\rangle=\sum_{j} B_{i j} I_{j}
$$

and a weighting function $W_{i j}$ defined as

$$
W_{i j}=\frac{B_{i j} I_{j}}{\sum_{j} B_{i j} I_{j}} .
$$

This weighting function is also normalized to unity within each beam:

$$
\sum_{j} W_{i j}=1
$$

A direct evaluation of equation (B3) would work but is very expensive, so we resort to a Taylor expansion.

\section{B2. TAYLOR EXPANSION}

At each frequency $v, Y$ depends only upon the $100 \mu \mathrm{m} / 240 \mu \mathrm{m}$ flux ratio, $\mathscr{R}$. We expand $Y(\mathscr{R})$ about the weighted mean ratio in FIRAS pixel $i, \overline{\mathscr{R}}_{i}$, as follows:

$$
Y\left(\mathscr{R}_{j}\right)=Y\left(\overline{\mathscr{R}}_{i}\right)+Y^{\prime}\left(\overline{\mathscr{R}}_{i}\right)\left(\mathscr{R}_{j}-\overline{\mathscr{R}}_{i}\right)+\frac{1}{2} Y^{\prime \prime}\left(\overline{\mathscr{R}}_{i}\right)\left[\mathscr{R}_{j}-\overline{\mathscr{R}}_{i}\right]^{2}+\frac{1}{6} Y^{\prime \prime \prime}\left(\overline{\mathscr{R}}_{i}\right)\left[\mathscr{R}_{j}-\overline{\mathscr{R}}_{i}\right]^{3}+\cdots,
$$

where the derivatives are with respect to $\mathscr{R}$ and we have dropped the $v$ subscript for clarity. Computing the weighted sum of $Y$ within 1 FIRAS pixel yields

$$
\sum_{j} W_{i j} Y\left(\mathscr{R}_{j}\right)=Y\left(\overline{\mathscr{R}}_{i}\right)+\frac{1}{2} Y^{\prime \prime}\left(\overline{\mathscr{R}}_{i}\right) \sigma_{i}^{2}(\mathscr{R})+\frac{1}{6} Y^{\prime \prime \prime}\left(\overline{\mathscr{R}}_{i}\right) s_{i}^{3}(\mathscr{R})+\cdots
$$

where the term linear in $\left(\mathscr{R}_{j}-\overline{\mathscr{R}}_{i}\right)$ vanishes, and

$$
\sigma_{i}^{2}(\mathscr{R})=\sum_{j} W_{i j}\left(\mathscr{R}_{j}-\overline{\mathscr{R}}_{i}\right)^{2}
$$

is a weighted variance within FIRAS pixel $i$ and

$$
\mathrm{s}_{i}^{3}(\mathscr{R})=\sum_{j} W_{i j}\left(\mathscr{R}_{j}-\overline{\mathscr{R}}_{i}\right)^{3}
$$

is a weighted difference cubed. Combining equations (B6) and (B11) the flux at any frequency is recovered via

$$
F_{i}(v)=\left\langle I_{i}\right\rangle\left[Y\left(\overline{\mathscr{R}}_{i}\right)+\frac{1}{2} Y^{\prime \prime}\left(\overline{\mathscr{R}}_{i}\right) \sigma_{i}^{2}(\mathscr{R})+\frac{1}{6} Y^{\prime \prime \prime}\left(\overline{\mathscr{R}}_{i}\right) s_{i}^{3}(\mathscr{R})\right] .
$$

Note that this expansion is implemented to describe temperature fluctuations within a FIRAS pixel. The values of $\left\langle I_{i}\right\rangle, \overline{\mathscr{R}}_{i}$, $\sigma_{i}^{2}(\mathscr{R})$, and $s_{i}^{3}(\mathscr{R})$ need be computed only once for all the DIRBE values within each FIRAS pixel. Once these values are saved, there is no need to return to the higher resolution DIRBE maps. The predictions for a given dust model establishes the relationship $Y(\mathscr{R})$, and the flux in FIRAS pixel $i$ is quickly computed via equation (B14).

We have carried the Taylor expansion to third order to establish convergence: the third-order terms are significantly smaller than the second-order terms and are usually negligible. All results in this paper are obtained with the third-order Taylor series. Setting $\sigma^{2}(\mathscr{R})$ equal to zero would ignore these small-scale temperature variations and would introduce errors at the level of a few percent in our model predictions.

\section{B3. DEFINITION OF $\chi^{2}$}

The comparison between predictions and the FIRAS data is further simplified by collapsing the problem spatially. At each frequency, a regression line is computed for the FIRAS flux as a function of the predicted flux (as in Fig. 2). The pixel weights from the FIRAS data are used for these regressions. The zero point of the best-fit slope is ignored, as it is sensitive to uncertainties in the zodiacal light model and the cosmic infrared background (CIB). The slope of the regression is our measure of goodness of fit for a model, with a slope of unity at all frequencies corresponding to perfect agreement. 
The $\chi^{2}$ for each model is computed from the 123 slope values and significance of their deviations from unity. The full covariance matrix $C_{i j}$ (FIRAS Exp. Supp. 1997, § 7.1.2) for the FIRAS data is used to couple the errors between frequency bins. We define a dimensionless covariance,

$$
C_{i j}^{\prime}=\frac{C_{i j}}{\sqrt{C_{i i} C_{j j}}},
$$

where $i, j$ index the 123 used frequency bins. The variance in the correlation slope $m$ at frequency $i, \sigma^{2}\left(m_{i}\right)$, is derived from the linear regression for each frequency, assuming uncorrelated Gaussian measurement noise. These $\sigma^{2}\left(m_{i}\right)$ values are dimensionless because the $m_{i}$ are dimensionless slopes of order unity. Because of the frequency covariance, the variance at frequency $i$ contains contributions from the measurement errors at all frequencies $j$ as

$$
\sigma^{2}\left(m_{i}\right)=\sum_{j} \sigma^{2}\left(m_{j}\right) C_{i j}^{\prime} .
$$

This covariance matrix does not include the contribution from the overall bolometer gain errors $J_{i}$ (termed JCJ errors in FIRAS Exp. Supp. 1997, § 7.3.2). The full covariance matrix includes the JCJ terms $J_{i} J_{j}$, yielding a $\chi^{2}$ of

$$
\chi^{2}=m_{i}\left[C_{i j}^{\prime} \sigma\left(m_{i}\right) \sigma\left(m_{j}\right)+J_{i} J_{j}\right]^{-1} m_{j} .
$$

This expression for $\chi^{2}$ is used for the fits in this paper.

\section{APPENDIX C}

\section{DATA PRESENTATION}

We provide an electronic data distribution that computes thermal emission from Galactic dust for any of the models considered in this paper. The preferred model is the two-component model with $\alpha_{1}=1.67, \alpha_{2}=2.70$. Intensities are computed at any frequency using equation (1) for single-component models and equation (6) for two-component models. The sky brightness is computed in units of MJy sr ${ }^{-1}$, which can be multiplied by $4024(v / 90 \mathrm{GHz})^{-2} \mu \mathrm{K}\left(\mathrm{MJy} \mathrm{sr}^{-1}\right)^{-1}$ to convert to a brightness temperature in $\mu \mathrm{K}$. Brightness temperature may be converted to thermodynamic $\Delta T$ by multiplying by the "Planckcorr" factor:

$$
\text { Planckcorr }=\frac{\left(e^{x}-1\right)^{2}}{x^{2} e^{x}},
$$

where $x=h v / k_{b} T_{\mathrm{CMB}}$ and $T_{\mathrm{CMB}}=2.73$. In Table 6, these factors are evaluated for a number of frequencies typical of CMB

\begin{tabular}{|c|c|c|c|c|}
\hline $\begin{array}{l}\text { Experiment } \\
\text { (1) }\end{array}$ & $\begin{array}{c}v \\
(\mathrm{GHz}) \\
(2)\end{array}$ & $\begin{array}{c}\lambda \\
(\mathrm{mm}) \\
(3)\end{array}$ & $\begin{array}{c}\text { Factor } \\
\mu \mathrm{K} /\left(\mathrm{MJy} \mathrm{sr}^{-1}\right) \\
\text { (4) }\end{array}$ & $\begin{array}{c}\text { Planckcorr } \\
\text { (5) }\end{array}$ \\
\hline \multirow[t]{3}{*}{$C O B E / \mathrm{DMR} . . .}$. & 31.5 & 9.52 & 32849 & 1.02582 \\
\hline & 53.0 & 5.66 & 11603 & 1.07448 \\
\hline & 90.0 & 3.33 & 4024 & 1.22684 \\
\hline \multirow[t]{5}{*}{$M A P \ldots \ldots \ldots \ldots$} & 22.0 & 13.64 & 67344 & 1.01253 \\
\hline & 30.0 & 10.00 & 36216 & 1.02340 \\
\hline & 40.0 & 7.50 & 20371 & 1.04190 \\
\hline & 60.0 & 5.00 & 9054 & 1.09623 \\
\hline & 90.0 & 3.33 & 4024 & 1.22684 \\
\hline \multirow[t]{9}{*}{ Planck .............. } & 30.0 & 10.00 & 36216 & 1.02340 \\
\hline & 44.0 & 6.82 & 16836 & 1.05087 \\
\hline & 70.0 & 4.29 & 6652 & 1.13275 \\
\hline & 100.0 & 3.00 & 3259 & 1.28562 \\
\hline & 143.0 & 2.10 & 1594 & 1.65110 \\
\hline & 217.0 & 1.38 & 692.2 & 2.98186 \\
\hline & 353.0 & 0.85 & 261.6 & 12.8186 \\
\hline & 545.0 & 0.55 & 109.7 & 157.85 \\
\hline & 857.0 & 0.35 & 44.38 & 15392 \\
\hline
\end{tabular}
anisotropy experiments.

TABLE 6

UNIT CONVERSION FACTORS FOR SELECTED CMBR EXPERIMENTS

Note-Col. (2): Frequency, in GHz, for which the unit conversion factors are computed. Col. (3): Corresponding wavelength in $\mathrm{mm}$. A value in units of MJy sr ${ }^{-1}$ should be multiplied by the factor in col. (4) to convert to $\mu \mathrm{K}$ brightness temperature. Brightness temperature is multiplied by Planckcorr (col. [5]; eq. [C1]) to convert to thermodynamic temperature, assuming $T_{\mathrm{CMB}}=2.73 \mathrm{~K}$. 
The predictions for thermal emission discussed in this paper are based upon the DIRBE $100 \mu \mathrm{m}$ map (with zodiacal light and CIB removed) and a ratio map, $\mathscr{R}$, which is a filtered $I_{100} / I_{240}$ flux ratio in the DIRBE passbands. These maps are stored as simple FITS images in pairs of $1024 \times 1024$ pixel Lambert ZEA (Zenithal Equal Area) polar projections, similar to the data format used for SFD98. The NGP projection covers the northern Galactic hemisphere, centered at $b=+90^{\circ}$, with latitude running clockwise. The SGP projection covers the southern Galactic hemisphere, centered at $b=-90^{\circ}$, with latitude running counterclockwise. (Note that Figs. 7, 8, 9, and 10 show the SGP projections rotated by $180^{\circ}$.) Galactic coordinates $(l$, $b)$ are converted to pixel positions $(x, y)$ via

$$
\begin{aligned}
& x=\frac{N}{2} \sqrt{1-n \sin (b)} \cos (l)+\frac{(N-1)}{2} \\
& y=-\frac{n N}{2} \sqrt{1-n \sin (b)} \sin (l)+\frac{(N-1)}{2},
\end{aligned}
$$

where $N=1024$ and $n=+1$ for the NGP, and $n=-1$ for the SGP. Pixel numbers are zero-indexed, with the center of the lower left pixel having position $(x, y)=(0,0)$. These Lambert projections are minimally distorted at high Galactic latitudes, with the distortion approaching $40 \%$ at $b=0^{\circ}$. The pixel area of $\left(9^{\prime} .49\right)^{2}$ oversamples the FWHM of $40^{\prime}$.

Predictions can be made at higher resolution by extrapolating from the IRAS rather than the DIRBE $100 \mu \mathrm{m}$ map. We use the high-resolution $100 \mu \mathrm{m}$ map from SFD98, which contains reprocessed IRAS/ISSA data recalibrated to DIRBE. These maps contain $4096 \times 4096$ pixels $(N=4096)$. The pixel size of $\left(2{ }^{\prime} \cdot 372\right)^{2}$ well samples the FWHM of $6^{\prime} 1$. This map has $\sim 20,000$ IRAS sources removed, which is appropriate for microwave predictions since IR-luminous stars and galaxies are not expected to contribute significantly to the microwave sky brightness.

The caveats to using these maps to predict emission from Galactic dust can be summarized as follows:

1. At frequencies on the Wien tail of the emission $(\lambda \lesssim 100 \mu \mathrm{m})$, we underestimate the dust emission by not including the contribution from small, transiently heated grains.

2. At microwave frequencies ( $v \lesssim 100 \mathrm{GHz}$ ), we have ignored magnetic dipole emission (cf. Draine \& Lazarian 1999) and electric dipole emission from rapidly rotating grains (cf. Draine \& Lazarian 1998b). Either of these mechanisms may be expected to dominate the thermal emission.

3. Our best-fit model is not a complete description of the dust properties. This model shows residuals that correlate with such environmental properties as the dust temperature and dust/gas ratio.

4. Unresolved infrared-luminous Galactic sources (primarily stars) are removed from the IRAS maps to a flux level of $f_{100} \approx 0.3 \mathrm{Jy}$ (see SFD98). These stars are not expected to contribute significantly to the sky brightness at frequencies $v \lesssim 1000$ $\mathrm{GHz}$, but this has not been explicitly tested.

5. Although the angular resolution is $40^{\prime}$ for the DIRBE $100 \mu \mathrm{m}$ map and 6.1 for the reprocessed IRAS $100 \mu \mathrm{m}$ map, our extrapolations to other frequencies relies on an $\mathscr{R}$ map with an effective resolution of 1.3 .

The data files and corresponding software will be available in the CD-ROM series of the AAS, or from our web site. ${ }^{4}$ Mask files are also available that contain the most important processing steps for any given position on the sky. Further details will be available with the data files.

\footnotetext{
${ }^{4} \mathrm{http}: / /$ astro.berkeley.edu/dust/index.html.
}

\section{REFERENCES}

Agladze, N. I., Sievers, A. J., Jones, S. A., Burlitch, J. M., \& Beckwith, S. V. W. 1996, ApJ, 462, 1026

Bennett, C. L., et al. 1996, ApJ, 646, L1

Blain, A. W., Kneib, J. P., Ivison, R. J., \& Smail, I. 1999, ApJ, 512, L87

Boulanger, F., Abergel, A., Bernard, J. P., Burton, W. B., Désert, F. X., Hartmann, D., Lagache, G., \& Puget, J. L. 1996, A\&A, 312, 256

COBE Diffuse Infrared Background Experiment (DIRBE) Explanatory Supplement. 1995, ed. M. G. Hauser, T. Kelsall, D. Leisawitz, \& Weiland, J. (COBE Ref. Pub. No. 95-A) (Greenbelt: NASA/GSFC)

$C O B E$ Far Infrared Absolute Spectrophotometer (FIRAS) Explanatory Supplement, Version 4. 1997, ed. S. Brodd, D. J. Fixsen, K. A. Jensen, J. C. Mather, \& R. A. Shafer (COBE Ref. Pub. No. 97-C) (Greenbelt: NASA/GSFC)

de Oliveira-Costa, A., Kogut, A., Devlin, M., Netterfield, C. B., Page, L. A., \& Wollack, E. J. 1997, ApJ, 482, L17

de Oliveira-Costa, A., Tegmark, M., Page, L., \& Boughn, S. 1998, ApJ, 509, L9

Draine, B. T., \& Anderson, N. 1985, ApJ, 292, 494

Draine, B. T., \& Lazarian, A. 1998a, ApJ, 494, L19 $1998 \mathrm{~b}$, ApJ, 508, 157 1999, ApJ, 512, 740

Draine, B. T., \& Lee, H. M. 1984, ApJ, 285, 89

Finkbeiner, D. P., Schlegel, D. J., \& Davis M. 1999, in preparation

Fixsen, D. J., et al. 1994, ApJ, 420, 457
Fixsen, D. J., Cheng, E. S., Gales, J. M., Mather, J. C., Shafer, R. A. \& Wright, E. L. 1996, ApJ, 473, 576

Fixsen, D. J., Mather, J. C., Shafer, R. A., Brodd, S., \& Jensen, K. A. 1997, AAS Meeting 191, 91.05

Fogel, M. E., \& Leung, C. M. 1998, ApJ, 501, 175

Hartmann, D., \& Burton, W. B. 1997, Atlas of Galactic Neutral Hydrogen (Cambridge: Cambridge Univ. Press)

Hauser, M. G., et al. 1998, ApJ, 508, 25

Hildebrand, R. H. 1983, QJRAS, 24, 267

Kim, S., Martin, P. G., \& Hendry, P. D. 1994, ApJ, 422, 164

Kittel, C. 1976, Introduction to Solid State Physics (New York: Wiley and Sons)

Kogut, A., et al. 1996, ApJ, 464, L5

Lagache, G., Abergel, A., Boulanger, F., Désert, F. X., \& Puget, J. L. 1999, A\&A, 344, 322L

Leitch, E. M., Readhead, A. C. S., Pearson, T. J., \& Myers, S. T. 1997, ApJ, 486, L23

Low, F. J., et al. 1984, ApJ, 278, L19

Mather, J. C. 1982, Opt. Eng., 21, 769

Mather, J. C., Fixsen, D. J., Shafer, R. A., Mosier, C. \& Wilkinson, D. T. 1999, ApJ, 512, 511

Mathis, J. S., Rumpl, W., \& Nordsieck, K. H. 1977, ApJ, 217, 425

Netterfield, C. B., Devlin, M. J., Jarosik, N., Page, L., \& Wollack, E. J. 1997, ApJ, 474, 47 
Netterfield, C. B., Jarosik, N., Page, L., Wilkinson, D., \& Wollack, E. 1995, ApJ, 445, L69

Pollack, J. B., Hollenbach, D., Beckwith, S., Simonelli, D. P., Roush, T., \& Fong, W. 1994, ApJ, 421, 615

Reach, W. T., et al. 1995, ApJ, 451, 188

Schlegel, D. J., Finkbeiner, D. P., \& Davis M. 1998, ApJ, 500, 525 (SFD98)
Smoot, G. F. et al. 1992, ApJ, 396, L1

Tielens, A. G. G. M., \& Allamandola, L. J. 1987, in Interstellar Processes, ed. D. J. Hollenbach \& H. A. Thronson (Dordrecht: Reidel), 397

Wheelock, S. L., et al. 1994, IRAS Sky Survey Atlas: Explanatory Supplement (JPL Publication 94-11) (Pasadena: JPL)

Wooten, B. J. 1972, Optical Properties of Solids (New York: Academic) 